TRANSACTIONS OF THE

AMERICAN MATHEMATICAL SOCIETY

Volume 356, Number 6, Pages 2349-2378

S 0002-9947(03)03400-7

Article electronically published on December 12, 2003

\title{
YOUNG WALL REALIZATION OF CRYSTAL BASES FOR CLASSICAL LIE ALGEBRAS
}

\author{
SEOK-JIN KANG, JEONG-AH KIM, HYEONMI LEE, AND DONG-UY SHIN
}

\begin{abstract}
In this paper, we give a new realization of crystal bases for finitedimensional irreducible modules over classical Lie algebras. The basis vectors are parameterized by certain Young walls lying between highest weight and lowest weight vectors.
\end{abstract}

\section{INTRODUCTION}

The classical Lie algebras and their representations have been the fundamental algebraic structure behind many branches of mathematics and mathematical physics. Throughout the past 100 years, it has been discovered that the representation theory of classical Lie algebras has a close connection with the combinatorics of Young tableaux and symmetric functions. (See, for example, 1], 15.) As can be found in [9], 16], this connection can be explained in a beautiful manner using the crystal basis theory for quantum groups, and one can derive many new and interesting results in combinatorial representation theory.

The quantum groups are deformations of the universal enveloping algebras of Kac-Moody algebras, and the crystal bases can be viewed as bases at $q=0$ for the integrable modules over quantum groups in the category $\mathcal{O}_{\text {int }}$. The crystal bases are given a structure of colored oriented graphs, called the crystal graphs, which reflect the combinatorial structure of integrable modules in the category $\mathcal{O}_{\text {int }}$. Moreover, they have many nice combinatorial features; for instance, they have a remarkably simple behavior with respect to taking the tensor product.

For classical Lie algebras, Kashiwara and Nakashima gave an explicit realization of crystal bases for finite-dimensional irreducible modules [9]. In their work, crystal bases were characterized as the sets of semistandard Young tableaux with given shapes satisfying certain additional conditions. Motivated by their work, Kang and Misra discovered a Young tableaux realization of crystal bases for finite-dimensional irreducible modules over the exceptional Lie algebra $G_{2}$ [5]. In [12, Littelmann gave another description of crystal bases for finite-dimensional simple Lie algebras using the Lakshmibai-Seshadri monomial theory. His approach was generalized (by himself) to the path model theory for all symmetrizable Kac-Moody algebras [13,

Received by the editors June 5, 2002 and, in revised form, April 2, 2003.

2000 Mathematics Subject Classification. Primary 81R50.

The first author's research was supported by KOSEF Grant \# 98-0701-01-5-L and the Young Scientist Award, Korean Academy of Science and Technology.

The second, third, and fourth authors' research was supported by KOSEF Grant \# 98-070101-5-L and BK21 Mathematical Sciences Division, Seoul National University. 
14]. Littelmann's theory also gives rise to colored oriented graphs, which turned out to be isomorphic to the crystal graphs [8].

In this paper, we give a new realization of crystal bases for finite-dimensional irreducible modules over classical Lie algebras. The basis vectors are parameterized by certain Young walls lying between the highest weight and lowest weight vectors. The Young walls were introduced in 2] and [3] as a combinatorial scheme for realizing the crystal bases for quantum affine algebras. They consist of colored blocks with various shapes built on the given ground-states and can be viewed as generalization of Young diagrams. The crystal bases for basic representations for quantum affine algebras are characterized as the sets of reduced proper Young walls 3 .

Let us briefly explain the main idea of our approach. Let $\mathfrak{g}$ be a classical Lie algebra lying inside an affine Lie algebra $\widehat{\mathfrak{g}}$ so that the Dynkin diagram of $\mathfrak{g}$ can be obtained by removing the 0 -node from the Dynkin diagram of $\widehat{\mathfrak{g}}$. Consider the crystal graph $B(\Lambda)$ of a basic representation $V(\Lambda)$ of $\widehat{\mathfrak{g}}$ consisting of reduced proper Young walls. If we remove all the 0 -arrows in $B(\Lambda)$, it is decomposed into a disjoint union of infinitely many connected components, each of which is isomorphic to the crystal graph $B(\lambda)$ for a finite-dimensional irreducible $\mathfrak{g}$-module $V(\lambda)$ with highest weight $\lambda$. Conversely, any crystal graph $B(\lambda)$ for a finite-dimensional irreducible $\mathfrak{g}$-module $V(\lambda)$ arises in this way. That is, given a dominant integral weight $\lambda$ for $\mathfrak{g}$, there is a dominant integral weight $\Lambda$ of level 1 for $\widehat{\mathfrak{g}}$ such that $B(\lambda)$ appears as a connected component in $B(\Lambda)$ without 0 -arrows.

Thus the remaining task is to characterize these connected components in $B(\Lambda)$. However, given a dominant integral weight $\lambda$ for the classical Lie algebra $\mathfrak{g}$, there are infinitely many connected components in $B(\Lambda)$ that are isomorphic to $B(\lambda)$. Among these, we choose the characterization of $B(\lambda)$ corresponding to the connected components having the least number of blocks.

In [10], from this Young wall realization of crystal bases over classical Lie algebras using affine combinatorial objects, Kim and Shin derived another tableaux realization, which is different from the one given by Kashiwara and Nakashima. Moreover, using the result of our work, Lee gave a realization of $A_{n}$-type Demazure crystals for certain highest weights 11 .

\section{Quantum groups And Young Walls}

The basic notions on quantum groups and crystal bases may be found in 2], 6, 7]. In this section, we mostly explain the basic combinatorics of Young walls which were introduced in [2, [3].

Let us fix basic notation:

$\mathfrak{g}$ : Kac-Moody algebra of finite classical type.

$U_{q}(\mathfrak{g})$ : quantum classical algebra.

$\widehat{\mathfrak{g}}$ : Kac-Moody algebra of affine type.

$U_{q}(\widehat{\mathfrak{g}})$ : quantum affine algebra.

$I$ : index set for simple roots of finite or affine Kac-Moody algebra.

$P^{\vee}=\left\{\begin{array}{ll}\bigoplus_{i \in I} \mathbf{Z} h_{i} & \text { for finite type } \\ \bigoplus_{i \in I} \mathbf{Z} h_{i} \oplus \mathbf{Z} d & \text { for affine type }\end{array}:\right.$ dual weight lattice.

$\alpha_{i}, \delta, \Lambda_{i}$ : simple root, null root, fundamental weight. 
$P=\left\{\lambda \in \mathfrak{h}^{*} \mid \lambda\left(P^{\vee}\right) \subset \mathbf{Z}\right\}$ : weight lattice.

$\tilde{e}_{i}, \tilde{f}_{i}:$ Kashiwara operators.

The Young walls are built of colored blocks with three different shapes:

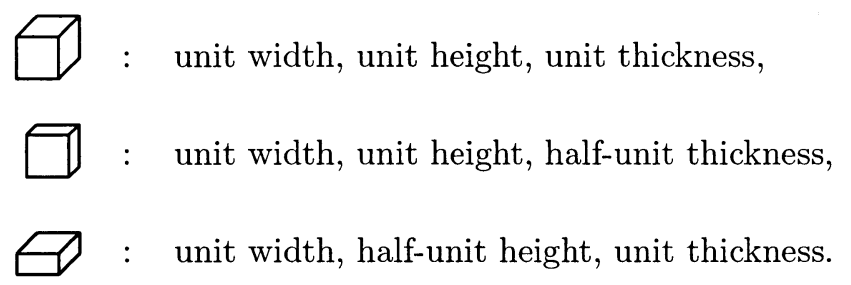

With these colored blocks, we build the walls of thickness less than or equal to 1 unit which extend infinitely to the left. Given a dominant integral weight $\Lambda$ of level 1 for the affine Lie algebra $\widehat{\mathfrak{g}}$, we fix a frame called the ground-state wall of weight $\Lambda$, and build the walls on this frame. For each type of quantum affine algebras, we use different sets of colored blocks and ground-state walls, whose description can be found in [2], 3].

The rules for building the walls are given as follows:

(1) The walls must be built on top of the ground-state wall.

(2) The colored blocks should be stacked in the patterns given in [2], [3].

(3) No block can be placed on top of a column of half-unit thickness.

(4) Except for the right-most column, there should be no free space to the right of any block.

By (4), the heights of the columns are weakly decreasing as we go from right to left. For this reason, the walls built by the above rules will be called the Young walls.

In the following example, for the affine Lie algebra $B_{n}^{(1)}$, we will illustrate the colored blocks, the ground-state wall, and the pattern for building the walls. For convenience, we will use the following notation:

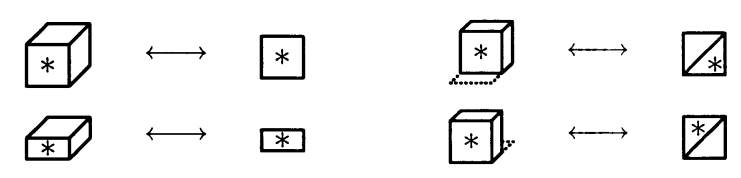

Example 1.1. The walls for the affine Lie algebra $B_{n}^{(1)}$ are built of the following data:

(a) Colored blocks:

$$
00,0, \pi, \quad(j=2, \cdots, n-1)
$$

(b) The ground-state wall of weight $\Lambda_{0}$ :

$$
Y_{\Lambda_{0}}=\begin{array}{|l|l|l|l|}
\hline 0 & 1 & 0 & 1 \\
\hline
\end{array}
$$


(c) The pattern for building the walls on $Y_{\Lambda_{0}}$ :

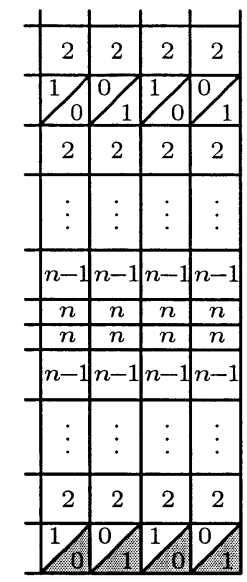

Definition 1.2. Let $\Lambda$ be a dominant integral weight of level 1 for the affine Lie algebra $\widehat{\mathfrak{g}}$.

(a) A column in a Young wall is called a full column if its height is a multiple of the unit length and its top is of unit thickness.

(b) For the classical quantum affine algebras of type $A_{2 n-1}^{(2)}(n \geq 3), D_{n}^{(1)}(n \geq 4)$, $A_{2 n}^{(2)}(n \geq 2), D_{n+1}^{(2)}(n \geq 2)$ and $B_{n}^{(1)}(n \geq 3)$, a Young wall is said to be proper if none of the full columns have the same height.

(c) For the quantum affine algebras of type $A_{n}^{(1)}(n \geq 1)$, every Young wall is defined to be proper.

Let $\delta$ be the null root for the quantum affine algebra $U_{q}(\widehat{\mathfrak{g}})$ and write

$$
\begin{cases}\delta=a_{0} \alpha_{0}+a_{1} \alpha_{1}+\cdots+a_{n} \alpha_{n} & \text { for } \widehat{\mathfrak{g}}=A_{n}^{(1)}, \cdots, B_{n}^{(1)}, \\ 2 \delta=a_{0} \alpha_{0}+a_{1} \alpha_{1}+\cdots+a_{n} \alpha_{n} & \text { for } \widehat{\mathfrak{g}}=D_{n+1}^{(2)} .\end{cases}
$$

The part of a column consisting of $a_{0}$-many 0-blocks, $a_{1}$-many 1 -blocks, $\cdots, a_{n^{-}}$ many $n$-blocks in some cyclic order is called a $\delta$-column.

Definition 1.3. (a) A column in a proper Young wall is said to contain a removable $\delta$ if we can remove a $\delta$-column from $Y$ and still obtain a proper Young wall.

(b) A proper Young wall is said to be reduced if none of its columns contain a removable $\delta$.

Let $\mathbf{F}(\Lambda)$ be the set of all proper Young walls and let $\mathbf{Y}(\Lambda)$ denote the set of all reduced proper Young walls. Then we can define a crystal structure on $\mathbf{F}(\Lambda)$ so that it may become a crystal graph for some integrable $U_{q}(\widehat{\mathfrak{g}})$-module in the category $\mathcal{O}_{\text {int }}$ [3], 4]. In this case, the set $\mathbf{Y}(\Lambda)$ becomes a connected component in the crystal graph $\mathbf{F}(\Lambda)$ and is isomorphic to the crystal graph $B(\Lambda)$ for the basic representation $V(\Lambda)$ of the quantum affine algebra $U_{q}(\widehat{\mathfrak{g}})$. We briefly explain the crystal structure of $\mathbf{F}(\Lambda)$. The main point is how to define the action of Kashiwara operators $\tilde{e}_{i}$ and $\tilde{f}_{i}(i=0,1, \cdots, n)$ on proper Young walls.

Definition 1.4. (a) A block of color $i$ in a proper Young wall is called a removable $i$-block if the wall remains a proper Young wall after removing the block. A column 
in a proper Young wall is called $i$-removable if the top of that column is a removable $i$-block.

(b) A place in a proper Young wall where one may add an $i$-block to obtain another proper Young wall is called an admissible $i$-slot. A column in a proper Young wall is called $i$-admissible if the top of that column is an admissible $i$-slot.

Fix $i \in I$ and let $Y=\left(y_{k}\right)_{k=0}^{\infty} \in \mathbf{F}(\Lambda)$ be a proper Young wall.

(1) To each column $y_{k}$ of $Y$, we assign its $i$-signature as follows:

(a) we assign - - if the column $y_{k}$ is twice $i$-removable; (the $i$-block will be of half-unit height in this case).

(b) we assign - if the column is once $i$-removable, but not $i$-admissible (the $i$-block may be of unit height or of half-unit height);

(c) we assign -+ if the column is once $i$-removable and once $i$-admissible (the $i$-block will be of half-unit height in this case);

(d) we assign + if the column is once $i$-admissible, but not $i$-removable (the $i$-block may be of unit height or of half-unit height);

(e) we assign ++ if the column is twice $i$-admissible (the $i$-block will be of half-unit height in this case).

(2) From the (infinite) sequence of +'s and -'s, cancel out every $(+,-)$-pair to obtain a finite sequence of -'s followed by + 's, reading from left to right. This sequence is called the $i$-signature of the proper Young wall $Y$.

(3) We define $\tilde{e}_{i} Y$ to be the proper Young wall obtained from $Y$ by removing the $i$-block corresponding to the right-most - in the $i$-signature of $Y$. We define $\tilde{e}_{i} Y=0$ if there exists no - in the $i$-signature of $Y$.

(4) We define $\tilde{f}_{i} Y$ to be the proper Young wall obtained from $Y$ by adding an $i$-block to the column corresponding to the left-most + in the $i$-signature of $Y$. We define $\tilde{f}_{i} Y=0$ if there exists no + in the $i$-signature of $Y$.

Then we have:

Theorem 1.5 ([3, 4]). (a) The set $\mathbf{F}(\Lambda)$ together with the Kashiwara operators defined as above becomes a crystal graph for an integrable $U_{q}(\widehat{\mathfrak{g}})$-module in the category $\mathcal{O}_{\text {int }}$.

(b) For all $i \in I$ and $Y \in \mathbf{Y}(\Lambda)$, we have

$$
\tilde{e}_{i} Y \in \mathbf{Y}(\Lambda) \cup\{0\} \quad \text { and } \quad \tilde{f}_{i} Y \in \mathbf{Y}(\Lambda) \cup\{0\} .
$$

Moreover, there exists a crystal isomorphism

$$
\mathbf{Y}(\Lambda) \stackrel{\sim}{\longrightarrow} B(\Lambda) \quad \text { given by } Y_{\Lambda} \longmapsto u_{\Lambda},
$$

where $u_{\Lambda}$ is the highest weight vector in $B(\Lambda)$.

\section{Realization of CRYSTAL bases}

In this section, we will state the main result of this paper - a new realization of crystal bases for finite-dimensional irreducible modules over classical Lie algebras.

Let us explain the main idea of our approach. Let $\mathfrak{g}$ be a classical Lie algebra lying inside an affine Lie algebra $\widehat{\mathfrak{g}}$ so that the Dynkin diagram of $\mathfrak{g}$ can be obtained by removing the 0 -node from the Dynkin diagram of $\widehat{\mathfrak{g}}$. In this paper, we will focus on the following pairs of a classical Lie algebra and an affine Lie algebra:

$$
A_{n} \subset A_{n}^{(1)}, C_{n} \subset A_{2 n-1}^{(2)}, B_{n} \subset B_{n}^{(1)}, D_{n} \subset D_{n}^{(1)} .
$$


Fix such a pair $\mathfrak{g} \subset \widehat{\mathfrak{g}}$ and let $\Lambda$ be a dominant integral weight of level 1 for the affine Lie algebra $\widehat{\mathfrak{g}}$. Then by Theorem 1.5, the crystal graph $B(\Lambda)$ is realized as the set $\mathbf{Y}(\Lambda)$ of all reduced proper Young walls built on the ground-state wall $Y_{\Lambda}$. If we remove all the 0 -arrows in $\mathbf{Y}(\Lambda)$, then it is decomposed into a disjoint union of infinitely many connected components, each of which is isomorphic to the crystal graph $B(\lambda)$ for some dominant integral weight $\lambda$ for $\mathfrak{g}$.

Conversely, any crystal graph $B(\lambda)$ for $\mathfrak{g}$ arises in this way. That is, given a dominant integral weight $\lambda$ for $\mathfrak{g}$, there is a dominant integral weight $\Lambda$ of level 1 for $\widehat{\mathfrak{g}}$ such that $B(\lambda)$ appears as a connected component in $B(\Lambda)$ without 0 arrows. More precisely, we denote by $\lambda_{i}(i=1, \cdots, n)$ and $\Lambda_{i}(i=0,1, \cdots, n)$ the fundamental weights for the quantum classical Lie algebras and the quantum affine algebras, respectively, and define the linear functionals $\omega_{i}$ by

1) $\mathfrak{g}=A_{n}, C_{n}:$

$$
\omega_{i}=\lambda_{i} \quad \text { for } i=1, \cdots, n
$$

2) $\mathfrak{g}=B_{n}$ :

$$
\omega_{i}= \begin{cases}\lambda_{i} & \text { for } i=1, \cdots, n-1 \\ 2 \lambda_{n} & \text { for } i=n\end{cases}
$$

3) $\mathfrak{g}=D_{n}$ :

$$
\omega_{i}= \begin{cases}\lambda_{i} & \text { for } i=1, \cdots, n-2, \\ \lambda_{n-1}+\lambda_{n} & \text { for } i=n-1, \\ 2 \lambda_{n} & \text { for } i=n, \\ 2 \lambda_{n-1} & \text { for } i=n+1 .\end{cases}
$$

Then for each dominant integral weight $\lambda$ for $\mathfrak{g}$, we may take the level 1 dominant integral weight $\Lambda$ for $\widehat{\mathfrak{g}}$ as follows:

1) $A_{n} \subset A_{n}^{(1)}$

$$
\begin{aligned}
& \lambda=a_{1} \omega_{1}+\cdots+a_{n} \omega_{n}, \\
& \Lambda=\Lambda_{i} \text { if } a_{1}+2 a_{2}+\cdots+n a_{n} \equiv i \bmod n+1,
\end{aligned}
$$

2) $C_{n} \subset A_{2 n-1}^{(2)}$

$$
\begin{aligned}
& \lambda=a_{1} \omega_{1}+\cdots+a_{n} \omega_{n}, \\
& \Lambda= \begin{cases}\Lambda_{0} & \text { if } a_{1}+2 a_{2}+\cdots+n a_{n} \text { is odd } \\
\Lambda_{1} & \text { if } a_{1}+2 a_{2}+\cdots+n a_{n} \text { is even }\end{cases}
\end{aligned}
$$

3) $B_{n} \subset B_{n}^{(1)}$

$$
\begin{aligned}
& \lambda=a_{1} \omega_{1}+\cdots+a_{n} \omega_{n}+b \lambda_{n}, \\
& \Lambda= \begin{cases}\Lambda_{0} & \text { if } b=0 \text { and } a_{1}+2 a_{2}+\cdots+n a_{n} \text { is odd } \\
\Lambda_{1} & \text { if } b=0 \text { and } a_{1}+2 a_{2}+\cdots+n a_{n} \text { is even } \\
\Lambda_{n} & \text { if } b=1\end{cases}
\end{aligned}
$$

4) $D_{n} \subset D_{n}^{(1)}$

$$
\lambda=a_{1} \omega_{1}+\cdots+a_{n+1} \omega_{n+1}+b_{1} \lambda_{n-1}+b_{2} \lambda_{n},
$$




$$
\Lambda= \begin{cases}\Lambda_{0} & \text { if } b_{1}=b_{2}=0 \text { and } a_{1}+2 a_{2}+\cdots+n a_{n}+n a_{n+1} \text { is odd } \\ \Lambda_{1} & \text { if } b_{1}=b_{2}=0 \text { and } a_{1}+2 a_{2}+\cdots+n a_{n}+n a_{n+1} \text { is even } \\ \Lambda_{n-1} & \text { if } b_{1}=1 \text { and } b_{2}=0 \\ \Lambda_{n} & \text { if } b_{1}=0 \text { and } b_{2}=1\end{cases}
$$

Now, we need to identify the highest weight vector $u_{\lambda}$ for $B(\lambda)$ with some reduced proper Young wall in $\mathbf{Y}(\Lambda)$ which is annihilated by all $\tilde{e}_{i}$ for $i=1, \cdots, n$. However, given a dominant integral weight $\lambda$ for $\mathfrak{g}$, there are infinitely many such Young walls in $\mathbf{Y}(\Lambda)$. Equivalently, given $\lambda$, there are infinitely many connected components of $\mathbf{Y}(\Lambda)$ without 0 -arrows that are isomorphic to $B(\lambda)$. Thus the main task is to characterize these connected components. Among these, we choose the characterization of $B(\lambda)$ corresponding to the connected components having the least number of blocks.

Given a dominant integral weight $\lambda$ for $\mathfrak{g}$, we describe an algorithm for constructing the highest weight vector $H_{\lambda}$ and lowest weight vector $L_{\lambda}$ inside $\mathbf{Y}(\Lambda)$. For our convenience, we will focus on the case of $\mathfrak{g}=B_{n}$ because this case contains all the characteristics of the remaining cases. If $\lambda=\omega_{i}(i=1, \cdots, n)$, let $H_{\omega_{i}}$ denote the Young wall (see Figure 1). Then it is easy to verify that $\tilde{e_{j}} H_{\omega_{i}}=0$ for all $j=1, \cdots, n$. That is, $H_{\omega_{i}}$ is a highest weight vector of weight $\omega_{i}$. For the lowest weight vector, we denote by $L_{\omega_{i}}$ the Young wall given in Figure 2.

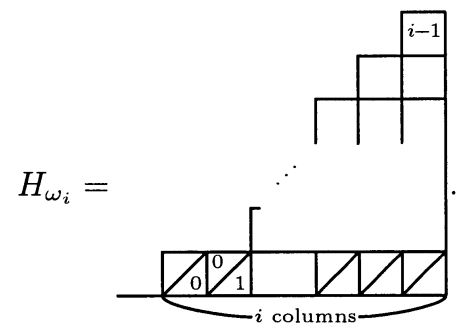

Figure 1.

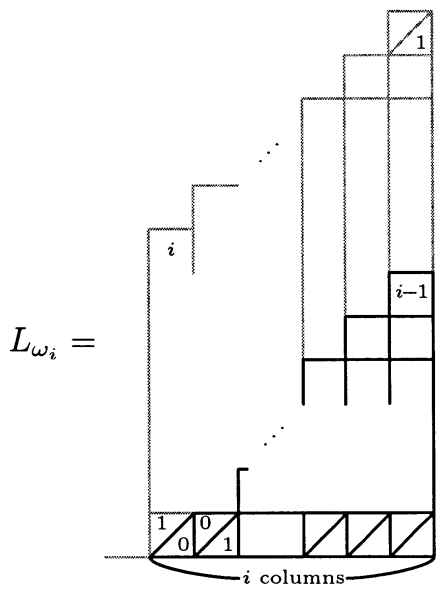

FIGURE 2. 


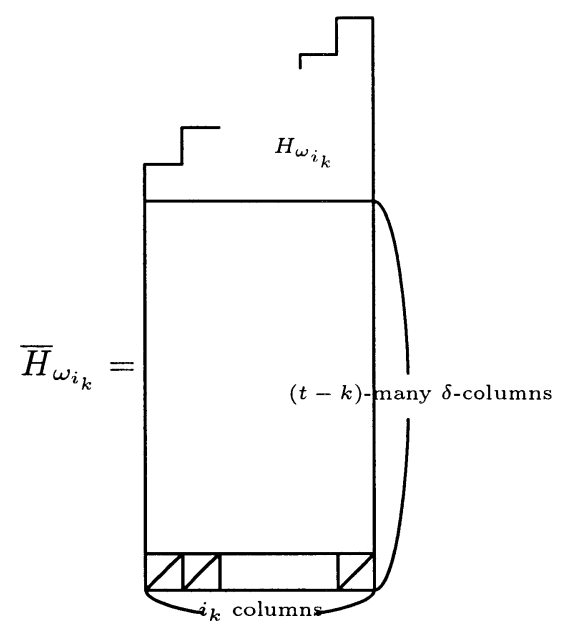

FiguRE 3.

Here, $H_{\omega_{i}}$ is denoted by the dark and bold-faced lines. Note that $\tilde{f}_{j} L_{\omega_{i}}=0$ for all $j=1, \cdots, n$. Thus $L_{\omega_{i}}$ is a lowest weight vector of weight $-\omega_{i}$. In Theorem 2.3. Theorem 2.8 Theorem 2.9 and Theorem 2.16, we will show that $L_{\omega_{i}}$ is in fact the lowest weight vector for the crystal graph $B\left(\omega_{i}\right)$; i.e., $L_{\omega_{i}}$ and $H_{\omega_{i}}$ are connected by Kashiwara operators.

If $\lambda=\lambda_{n}$, then the highest weight vector $H_{\lambda_{n}}$ and the lowest weight vector $L_{\lambda_{n}}$ for $B\left(\lambda_{n}\right)$ are given by

$$
H_{\lambda_{n}}=\underset{\cdots|n| n|\perp| n|n|}{ }
$$

and

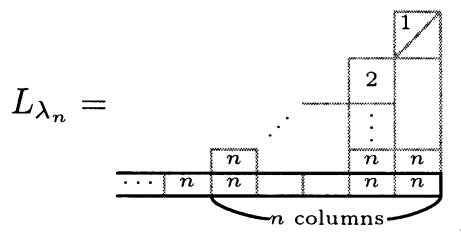

Here, $H_{\lambda_{n}}$ is denoted by the dark and bold-faced lines.

Suppose $\lambda$ has the form $\lambda=\omega_{i_{1}}+\cdots+\omega_{i_{t}}\left(1 \leq i_{1} \leq \cdots \leq i_{t} \leq n\right)$. For each $k=1, \cdots, t$, let $\bar{H}_{\omega_{i_{k}}}$ (resp. $\bar{L}_{\omega_{i_{k}}}$ ) denote the Young wall consisting of $H_{\omega_{i_{k}}}$ (resp. $\left.L_{\omega_{i_{k}}}\right)$ and $i_{k} \times(t-k)$-many $\delta$-columns. Here, we place $H_{\omega_{i_{k}}}$ (resp. $L_{\omega_{i_{k}}}$ ) on top of $\delta$-columns as is shown in Figure 3.

We define $H_{\lambda}$ (resp. $L_{\lambda}$ ) to be the Young wall obtained by attaching $\bar{H}_{\omega_{i_{k+1}}}$ (resp. $\bar{L}_{\omega_{i_{k+1}}}$ ) to the left-hand side of $\bar{H}_{\omega_{i_{k}}}\left(\right.$ resp. $\bar{L}_{\omega_{i_{k}}}$ ) for $k=1, \cdots, t-1$.

On the other hand, suppose $\lambda$ has the form $\lambda=\omega_{i_{1}}+\cdots+\omega_{i_{t}}+\lambda_{n}\left(1 \leq i_{1} \leq\right.$ $\left.\cdots \leq i_{t} \leq n\right)$. For each $k=1, \cdots, t$, let $\bar{H}_{\omega_{i_{k}}}$ (resp. $\bar{L}_{\omega_{i_{k}}}$ ) denote the Young wall consisting of $H_{\omega_{i_{k}}}$ (resp. $\left.L_{\omega_{i_{k}}}\right)$ and $i_{k} \times\left(t-k+\frac{1}{2}\right)$-many $\delta$-columns (see Figure 4 ).

We define $H_{\lambda}$ (resp. $L_{\lambda}$ ) to be the Young wall obtained by attaching $\bar{H}_{\omega_{i_{k+1}}}$ (resp. $\bar{L}_{\omega_{i_{k+1}}}$ ) to the left-hand side of $\bar{H}_{\omega_{i_{k}}}$ (resp. $\bar{L}_{\omega_{i_{k}}}$ ) and $H_{\lambda_{n}}$ (resp. $L_{\lambda_{n}}$ ) to the left-hand side of $\bar{H}_{\omega_{i_{t}}}$ (resp. $\left.\bar{L}_{\omega_{i_{t}}}\right)$. 


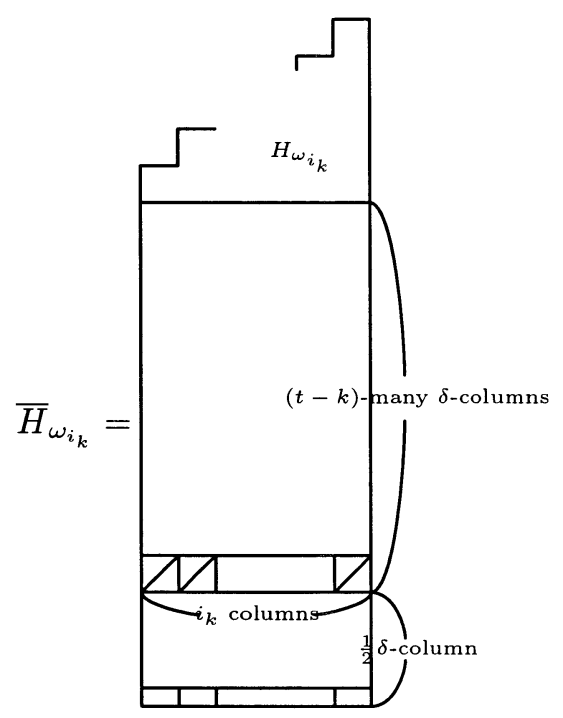

FiguRE 4.

Example 2.1. In this example, we will give descriptions of $H_{\lambda}$ and $L_{\lambda}$ for various choices of dominant integral weights $\lambda$ for $\mathfrak{g}=B_{3}$. The highest weight vector $H_{\lambda}$ will be denoted by the dark, bold-faced lines and the lowest weight vector $L_{\lambda}$ will be denoted by the bright, dotted lines.

(a) If $\lambda=\omega_{1}$, we choose $\Lambda=\Lambda_{1}$ and if $\lambda=\omega_{2}$, we choose $\Lambda=\Lambda_{0}$. The vectors $H_{\lambda}$ and $L_{\lambda}$ are given by:
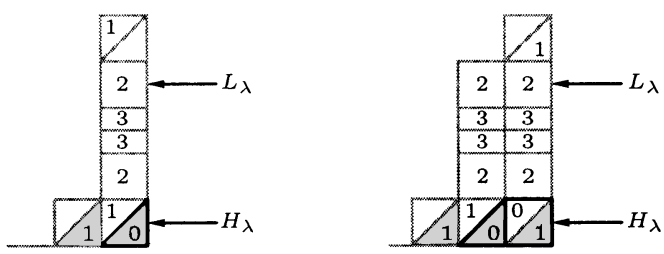

(b) If $\lambda=\omega_{3}$, we choose $\Lambda=\Lambda_{1}$, and if $\lambda=\omega_{1}+\omega_{3}$, we choose $\Lambda=\Lambda_{0}$. The vectors $H_{\lambda}$ and $L_{\lambda}$ are given by:
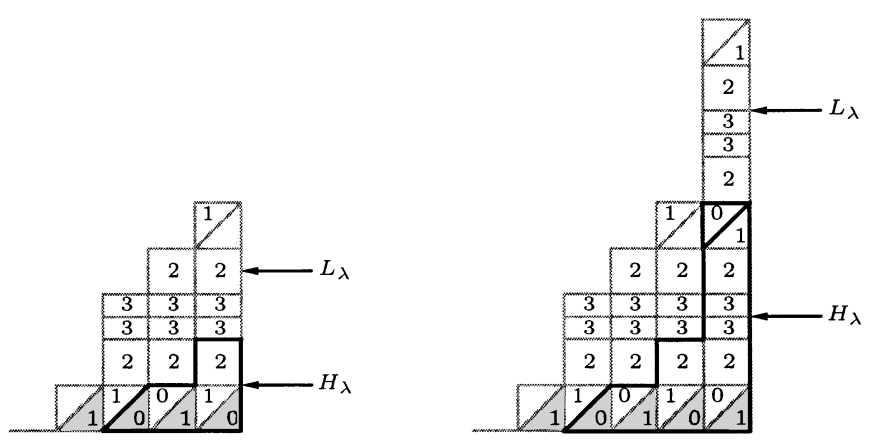
(c) If $\lambda=\omega_{1}+\lambda_{3}$ or $\omega_{2}+\lambda_{3}$, we choose $\Lambda=\Lambda_{3}$. The vectors $H_{\lambda}$ and $L_{\lambda}$ are given by:
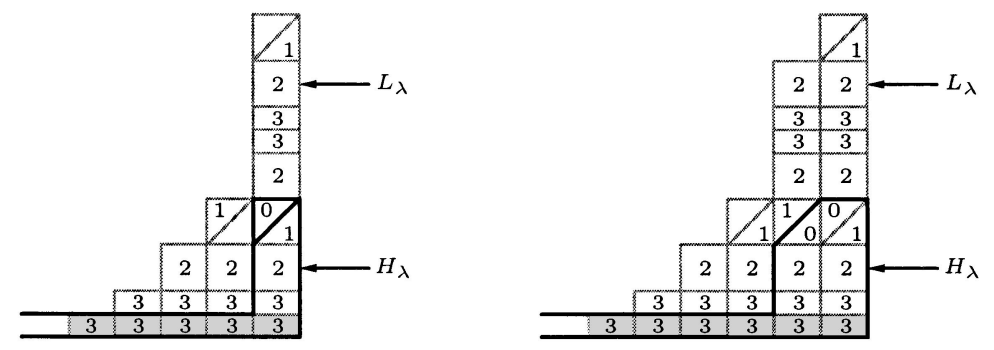

We now begin to characterize the crystal graph $B(\lambda)$ inside $\mathbf{Y}(\Lambda)$. Let $F(\lambda)$ denote the set of all reduced proper Young walls lying between $H_{\lambda}$ and $L_{\lambda}$. To describe $B(\lambda)$ inside $F(\lambda)$, we need some additional conditions. For this purpose, we need to introduce some notation. Fix a dominant integral weight $\lambda$ as follows:

$$
\lambda= \begin{cases}\omega_{i_{1}}+\cdots+\omega_{i_{t}} & \text { if } \mathfrak{g}=A_{n}, C_{n} \\ \omega_{i_{1}}+\cdots+\omega_{i_{t}}+b \lambda_{n} & \text { if } \mathfrak{g}=B_{n} \\ \omega_{i_{1}}+\cdots+\omega_{i_{t}}+b_{1} \lambda_{n-1}+b_{2} \lambda_{n} & \text { if } \mathfrak{g}=D_{n}\end{cases}
$$

where $b=0$ or $1,\left(b_{1}, b_{2}\right)=(1,0)$ or $(0,1)$.

For each $Y \in F(\lambda)$, we denote by $\stackrel{\circ}{Y}_{\omega_{i_{k}}}(k=1, \cdots, t)$ (resp. $\left.\stackrel{\circ}{Y}_{\lambda_{n-1}}, \stackrel{\circ}{Y}_{\lambda_{n}}\right)$ the part of $Y$ consisting of the blocks lying above $\bar{H}_{\omega_{i_{k}}}$ (resp. $H_{\lambda_{n-1}}, H_{\lambda_{n}}$ ) and we denote by $\bar{Y}_{\omega_{i_{k}}}$ (resp. $\bar{Y}_{\lambda_{n-1}}, \bar{Y}_{\lambda_{n}}$ ) the intersection of $Y$ and $\bar{L}_{\omega_{i_{k}}}$ (resp. $L_{\lambda_{n-1}}$, $L_{\lambda_{n}}$ ) as is shown in Figure 5. Moreover, we denote by $\bar{Y}_{\omega_{i_{k}}+\omega_{i_{k+1}}}$ (resp. $\bar{Y}_{\omega_{i_{t}}+\lambda_{n-1}}$, $\left.\bar{Y}_{\omega_{i_{t}}+\lambda_{n}}\right)$ the union of $\bar{Y}_{\omega_{i_{k}}}$ (resp. $\left.\bar{Y}_{\omega_{i_{t}}}\right)$ and $\bar{Y}_{\omega_{i_{k+1}}}\left(\operatorname{resp} . \bar{Y}_{\lambda_{n-1}}\right.$ or $\left.\bar{Y}_{\lambda_{n}}\right)$.

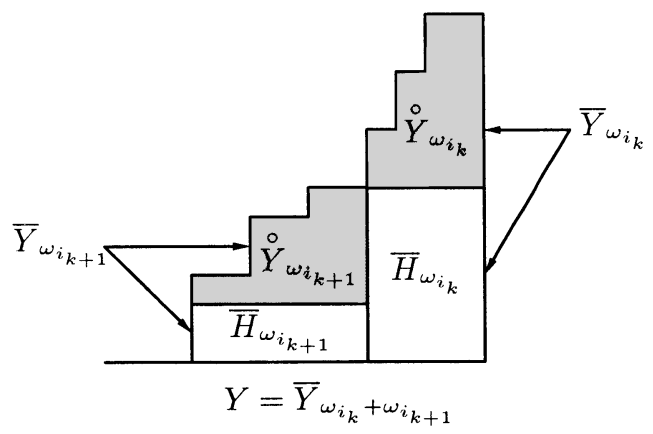

FIGURE 5 .

Now, consider $\bar{Y}_{\omega_{i_{k}}+\omega_{i_{k+1}}}, \bar{Y}_{\omega_{i_{t}}+\lambda_{n-1}}$ and $\bar{Y}_{\omega_{i_{t}}+\lambda_{n}}$ of $Y$. Then we define

$$
\begin{aligned}
& Y^{\omega_{i_{k}}}=\stackrel{\circ}{Y}_{\omega_{i_{k}}} \cap \bar{L}_{\omega_{i_{k+1}}} \quad \text { reading from top to bottom, } \\
& Y^{\omega_{i_{k+1}}}=\stackrel{\circ}{Y}{ }_{\omega_{i_{k+1}}} \cap \bar{L}_{\omega_{i_{k}}} \quad \text { reading from right to left in } \bar{Y}_{\omega_{i_{k}}+\omega_{i_{k+1}}} .
\end{aligned}
$$


Similarly, we define

$$
\begin{aligned}
& Y^{\omega_{i_{t}}}=\stackrel{\circ}{Y}_{\omega_{i_{t}}} \cap L_{\lambda_{n-1}}, \quad Y^{\lambda_{n-1}}=\stackrel{\circ}{Y}_{\lambda_{n-1}} \cap \bar{L}_{\omega_{i_{t}}} \quad \text { in } \bar{Y}_{\omega_{i_{t}}+\lambda_{n-1}}, \\
& Y^{\omega_{i_{t}}}=\stackrel{\circ}{Y}_{\omega_{i_{t}}} \cap L_{\lambda_{n}}, \quad Y^{\lambda_{n}}=\stackrel{\circ}{Y}_{\lambda_{n}} \cap \bar{L}_{\omega_{i_{t}}} \text { in } \bar{Y}_{\omega_{i_{t}}+\lambda_{n}} .
\end{aligned}
$$

Example 2.2. If $\mathfrak{g}=A_{4}, \lambda=\omega_{2}+\omega_{3}$, and

$$
Y=\begin{array}{|l|l|l|l|l|}
\multicolumn{1}{c}{} & & & 4 \\
\hline & 1 & 3 & 2 \\
\hline & 3 & 4 & 0 & 1 \\
\hline 1 & 2 & 3 & 4 & 0 \\
\hline
\end{array}
$$

then we have

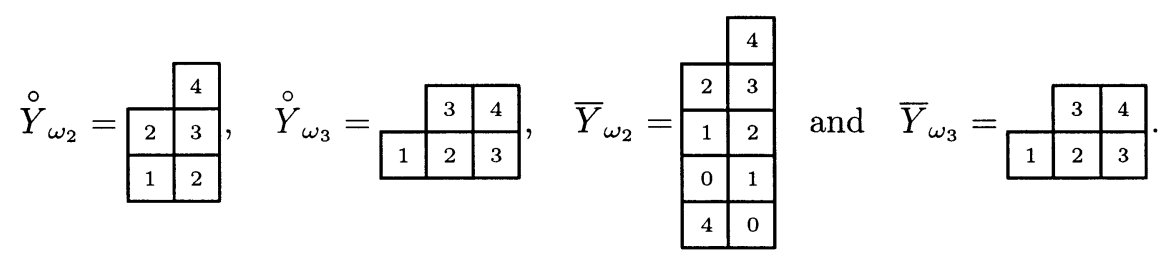

Moreover, we have

$$
Y^{\omega_{2}}=\begin{array}{|l|l|}
\cline { 2 - 2 } & 4 \\
\hline 2 & 3 \\
\hline
\end{array} \text { and } Y^{\omega_{3}}=\begin{array}{|l|l|}
\hline 3 & 4 \\
\hline 2 & 3 \\
\hline
\end{array}
$$

With this notation, we are ready to give an explicit description of the crystal graph $B(\lambda)$ over $\mathfrak{g}=A_{n}$.

Theorem 2.3. Let $\lambda \in P^{+}$be a dominant integral weight and write

$$
\lambda=\omega_{i_{1}}+\cdots+\omega_{i_{t}} \quad\left(1 \leq i_{1} \leq \cdots \leq i_{t} \leq n\right) .
$$

Set

$$
Y(\lambda)=\left\{Y \in F(\lambda) \mid Y^{\omega_{i_{k}}} \subset Y^{\omega_{i_{k+1}}} \text { in } \bar{Y}_{\omega_{i_{k}}+\omega_{i_{k+1}}} \text { for all } k=1,2, \cdots, t-1\right\} .
$$

Then there exists an isomorphism of $U_{q}\left(A_{n}\right)$-crystals

$$
Y(\lambda) \stackrel{\sim}{\longrightarrow} B(\lambda) \quad \text { given by } H_{\lambda} \longmapsto u_{\lambda},
$$

where $u_{\lambda}$ is the highest weight vector in $B(\lambda)$.

Example 2.4. Let $\mathfrak{g}=A_{4}$ and $\lambda=\omega_{2}+\omega_{3}$. For each Young wall given in Figure 6 , the shaded part represents $Y^{\omega_{2}}$ and $Y^{\omega_{3}}$, respectively. Hence, by Theorem 2.3 the first Young wall belongs to $Y(\lambda)$, but the second one does not.

Next, we will consider the case when $\mathfrak{g}=C_{n}$ or $B_{n}$. Consider $\bar{Y}_{\omega_{i_{k}}}$ for $k=$ $1, \cdots, t$. Suppose that $\bar{Y}_{\omega_{i_{k}}}$ contains a row consisting of $n$-blocks, which will be called the $n$-row, as is shown in Figure 7. 

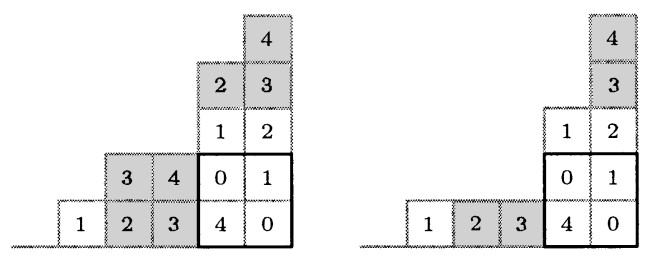

Figure 6.
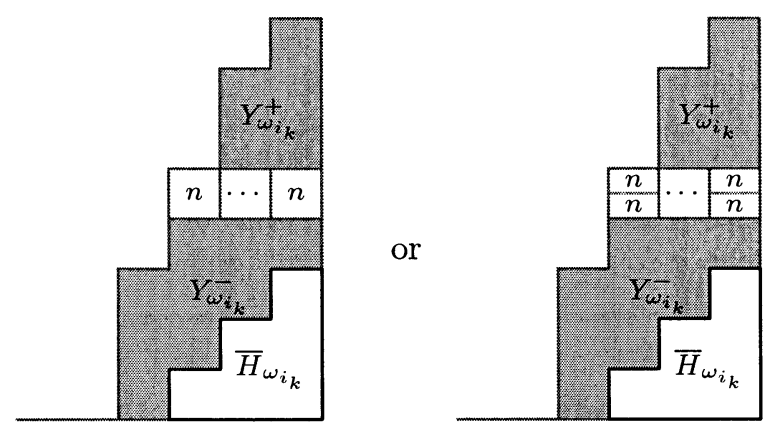

\section{FiguRE 7.}

We will denote by $Y_{\omega_{i_{k}}}^{+}\left(\right.$resp. $\left.Y_{\omega_{i_{k}}}^{-}\right)$the part of $Y$ consisting of the blocks lying above (resp. below) the $n$-row and below $\bar{L}_{\omega_{i_{k}}}$ (resp. above $\bar{H}_{\omega_{i_{k}}}$ ). We also denote by $\left|Y_{\omega_{i_{k}}}^{-}\right|$the wall obtained by reflecting $Y_{\omega_{i_{k}}}^{-}$along the $n$-row and shifting the blocks to the right as much as possible.

Example 2.5. If $\mathfrak{g}=C_{4}, \lambda=\omega_{4}$, and

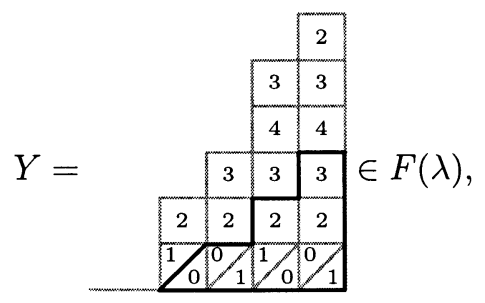

then we have

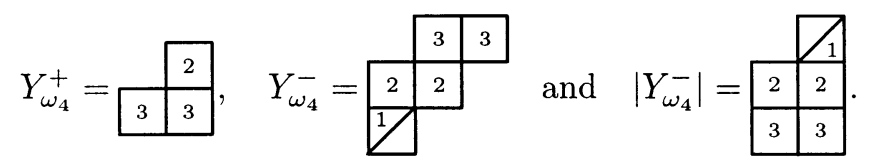

Now, consider $\bar{L}_{\omega_{i_{k}}+\omega_{i_{k+1}}}(k=1, \cdots, t-1)$ and $\bar{L}_{\omega_{i_{t}}+\lambda_{n}}$ (see Figure 8). As we can see, $\bar{L}_{\omega_{i_{k}}+\omega_{i_{k+1}}}$ and $\bar{L}_{\omega_{i_{t}}+\lambda_{n}}$ contain two $n$-rows above $\bar{H}_{\omega_{i_{k}}+\omega_{i_{k+1}}}$. Note that there are $i$-many blocks in the upper $n$-row. Let us denote by $b_{L}$ (resp. $b_{R}$ ) the left-most (resp. right-most) block in the upper $n$-row. Then the blocks $b_{L}, b_{R}$ and the block $b$ lying in the $(i-1)$-th row below $b_{L}$ form a right isosceles triangle. We denote by $L_{\left(\omega_{i_{k}}, \omega_{i_{k+1}}\right)}^{-}\left(\right.$resp. $\left.L_{\left(\omega_{i_{t}}, \lambda_{n}\right)}^{-}\right)$the part of $\bar{L}_{\omega_{i_{k}}+\omega_{i_{k+1}}}\left(\right.$ resp. $\bar{L}_{\omega_{i_{t}}+\lambda_{n}}$ ) constituting this right isosceles triangle. 


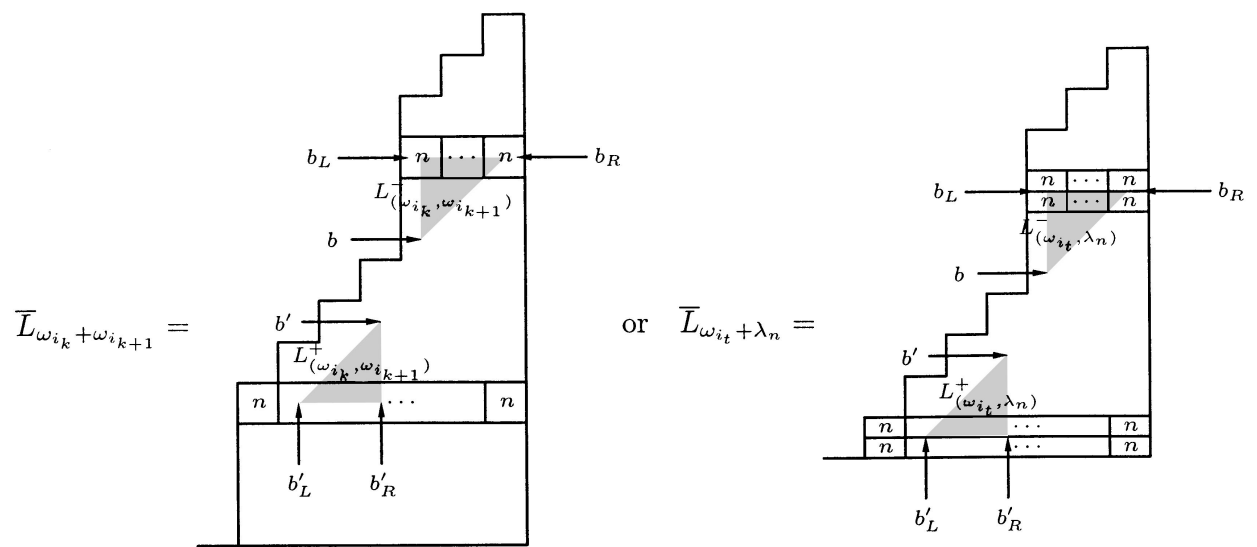

FiguRe 8.

Similarly, let $b_{R}^{\prime}$ be the right-most block in the lower $n$-row outside the highest weight vector $\bar{H}_{\omega_{i_{k}}+\omega_{i_{k+1}}}$ and $\bar{H}_{\omega_{i_{t}}+\lambda_{n}}$ and let $b_{L}^{\prime}$ be the $n$-block lying in the $(i-1)$ th column to the left of $b_{R}^{\prime}$. Then $b_{R}^{\prime}, b_{L}^{\prime}$ and the block $b^{\prime}$ lying in the $(i-1)$-th row above $b_{R}^{\prime}$ form another right isosceles triangle. We denote by $L_{\left(\omega_{i_{k}}, \omega_{i_{k+1}}\right)}^{+}$(resp. $\left.L_{\left(\omega_{i}, \lambda_{n}\right)}^{+}\right)$the part of $\bar{L}_{\omega_{i_{k}}+\omega_{i_{k+1}}}$ (resp. $\bar{L}_{\omega_{i_{t}}+\lambda_{n}}$ ) constituting this right isosceles triangle. Note that $L_{\left(\omega_{i_{k}}, \omega_{i_{k+1}}\right)}^{-}\left(\right.$resp. $\left.L_{\left(\omega_{i_{t}}, \lambda_{n}\right)}^{-}\right)$and $L_{\left(\omega_{i_{k}}, \omega_{i_{k+1}}\right)}^{+}\left(\operatorname{resp.} L_{\left(\omega_{i_{t}}, \lambda_{n}\right)}^{+}\right)$ are of the same size with each base of length $i$. Now, for each $Y \in F(\lambda)$, set

$$
\begin{aligned}
& Y_{\left(\omega_{i_{k}}, \omega_{i_{k+1}}\right)}^{-}=Y \cap L_{\left(\omega_{i_{k}}, \omega_{i_{k+1}}\right)}^{-}, \quad Y_{\left(\omega_{i_{k}}, \omega_{i_{k+1}}\right)}^{+}=Y \cap L_{\left(\omega_{i_{k}}, \omega_{i_{k+1}}\right)}^{+}, \\
& Y_{\left(\omega_{i_{t}}, \lambda_{n}\right)}^{-}=Y \cap L_{\left(\omega_{i_{t}}, \lambda_{n}\right)}^{-}, \quad Y_{\left(\omega_{i_{t}}, \lambda_{n}\right)}^{+}=Y \cap L_{\left(\omega_{i_{t}}, \lambda_{n}\right)}^{+}
\end{aligned}
$$

and denote by $\left|Y_{\left(\omega_{i_{k}}, \omega_{i_{k+1}}\right)}^{-}\right|$(resp. $\left.\left|Y_{\left(\omega_{i_{t}}, \lambda_{n}\right)}^{-}\right|\right)$the wall obtained by reflecting $Y_{\left(\omega_{\left.i_{k}, \omega_{i_{k+1}}\right)}\right.}^{-}\left(\right.$resp. $\left.Y_{\left(\omega_{i_{t}}, \lambda_{n}\right)}^{-}\right)$with respect to the upper $n$-row and shifting the blocks to the right as much as possible.

Example 2.6. If $\mathfrak{g}=B_{5}, \lambda=\omega_{3}+\lambda_{5}$ and

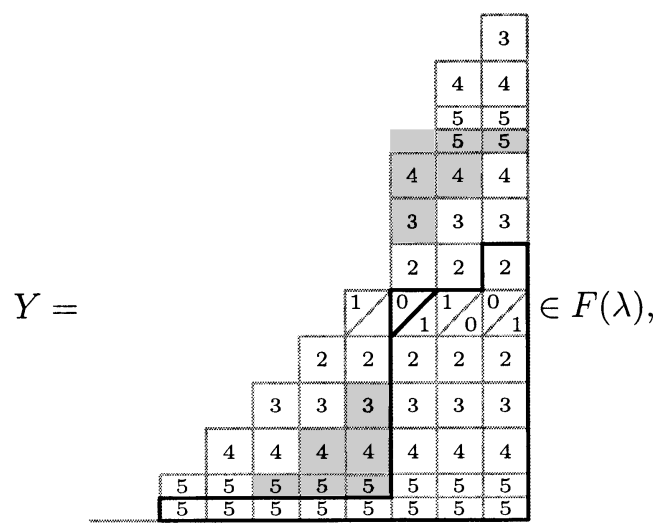


then we have

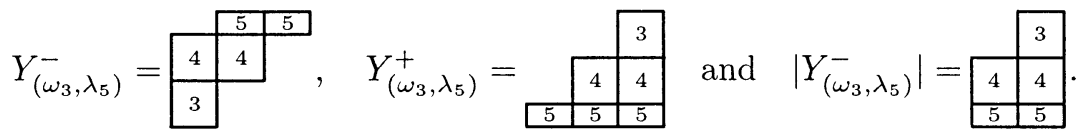

Here, the shaded parts represent $L_{\left(\omega_{3}, \lambda_{5}\right)}^{-}$and $L_{\left(\omega_{3}, \lambda_{5}\right)}^{+}$.

For $a=1, \cdots, n-1$, consider the $\bar{Y}_{\omega_{i_{k}}+\omega_{i_{k+1}}}$ and $\bar{Y}_{\omega_{i_{t}}+\lambda_{n}}$ of $Y \in F(\lambda)$ having the configuration in Figure 9. That is, the top of the $p$-th column of $\bar{Y}_{\omega_{i_{k}}}$ (resp. $\bar{Y}_{\omega_{i_{t}}}$ ) from the right is $\frac{a-1}{\frac{a-2}{a}}$ and the top of the $q$-th column of $\bar{Y}_{\omega_{i_{k+1}}}$ (resp. $\bar{Y}_{\lambda_{n}}$ ) from the right is $\frac{a}{a+1}$ with $p>q$.

(C1)

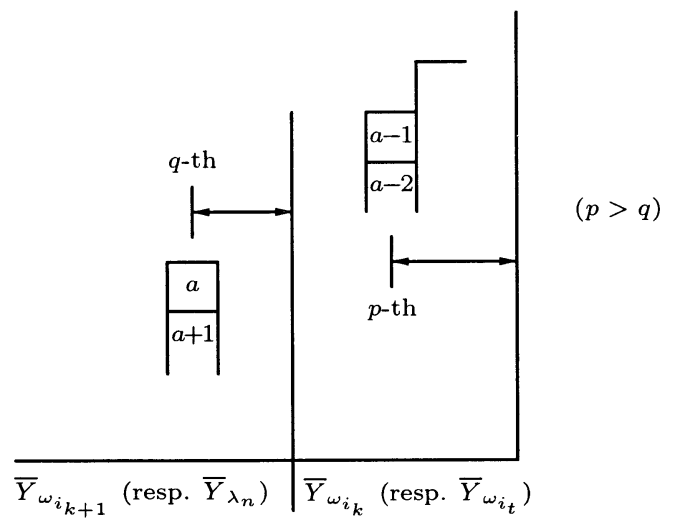

FIGURE 9 .

We define $L_{\omega_{i_{k}}}^{+}(a ; p, q)$ (resp. $\left.L_{\omega_{i_{k+1}}}^{+}(a ; p, q)\right)$ to be the right isosceles triangle formed by an $a$-block in the $q$-th column, an $(a+p-q-1)$-block in the $q$-th column and an $(a+p-q-1)$-block in the $(p-1)$-th column in $Y_{\omega_{i_{k}}}$ (resp. $\left.Y_{\omega_{i_{k+1}}}\right)$. Then the wall obtained by reflecting $L_{\omega_{i_{k}}}^{+}(a ; p, q)$ (resp. $\left.L_{\omega_{i_{k+1}}}^{+}(a ; p, q)\right)$ with respect to the $n$-row will be denoted by $L_{\omega_{i_{k}}}^{-}(a ; p, q)$ (resp. $\left.L_{\omega_{i_{k+1}}}^{-}(a ; p, q)\right)$. The shaded parts in Figure 10 represent $L_{\omega_{i_{k}}}^{ \pm}(a ; p, q)$ and $L_{\omega_{i_{k+1}}}^{ \pm}(a ; p, q)$. Now, we also define $L_{\omega_{i_{t}}}^{ \pm}(a, p, q)$ in a similar way, and for each $Y \in F(\lambda)$, set

$$
\begin{aligned}
& Y_{\omega_{i_{k}}}^{ \pm}(a ; p, q)=L_{\omega_{i_{k}}}^{ \pm}(a ; p, q) \cap Y, \quad Y_{\omega_{i_{k+1}}}^{ \pm}(a ; p, q)=L_{\omega_{i_{k+1}}}^{ \pm}(a ; p, q) \cap Y, \\
& Y_{\omega_{i_{t}}}^{ \pm}(a ; p, q)=L_{\omega_{i_{t}}}^{ \pm}(a ; p, q) \cap Y,
\end{aligned}
$$

and let $\left|Y_{\omega_{i_{k}}}^{-}(a ; p, q)\right|$ be the wall obtained by reflecting $Y_{\omega_{i_{k}}}^{-}(a ; p, q)$ with respect to the $n$-row and shifting the blocks to the right as much as possible. 


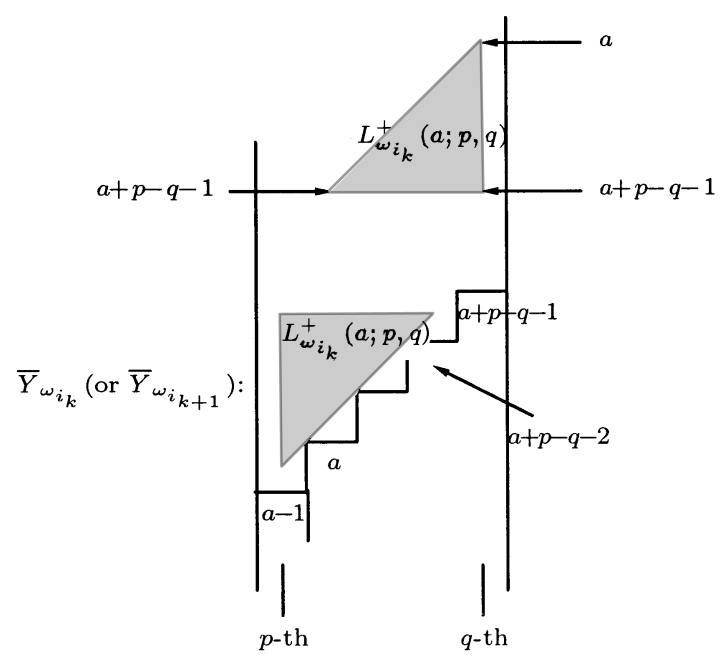

FiguRe 10.

Example 2.7. If $\mathfrak{g}=C_{5}, \lambda=\omega_{3}+\omega_{4}$ and

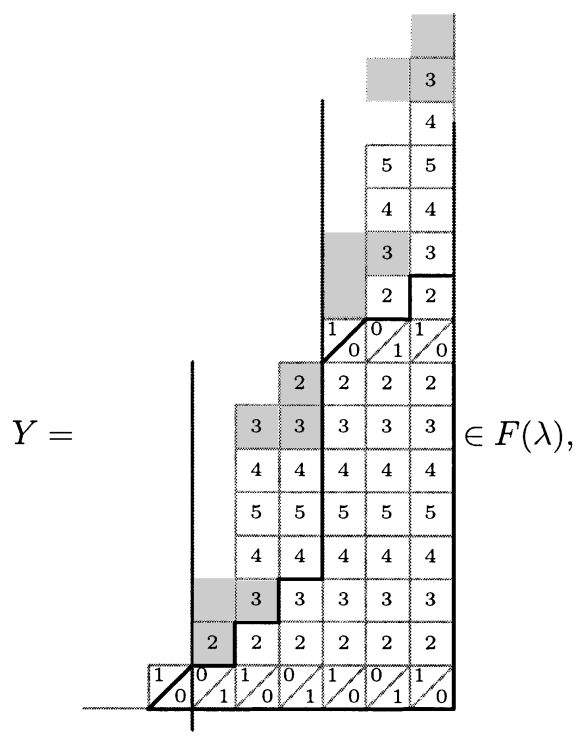

then we have

$$
\begin{aligned}
& Y_{\omega_{3}}^{ \pm}(2 ; 3,1)=\left|Y_{\omega_{3}}^{-}(2 ; 3,1)\right|=3 \\
& Y_{\omega_{4}}^{+}(2 ; 3,1)=\begin{array}{l|l|l|}
\hline & 2 \\
\hline 3 & 3
\end{array}, \quad Y_{\omega_{4}}^{-}(2 ; 3,1)=\begin{array}{|l|l}
3 & 3 \\
\hline &
\end{array}
\end{aligned}
$$

and

$$
\left|Y_{\omega_{4}}^{-}(2 ; 3,1)\right|=\frac{2}{3} .
$$

Here, the shaded parts represent $L_{\omega_{i}}^{ \pm}(2 ; 3,1)$ for $i=3,4$. 
Now, we are ready to give an explicit description of the crystal graph $B(\lambda)$ over $\mathfrak{g}=C_{n}$ and $B_{n}$.

Theorem 2.8. Let $\lambda \in P^{+}$be a dominant integral weight for $\mathfrak{g}=C_{n}$, and write

$$
\lambda=\omega_{i_{1}}+\cdots+\omega_{i_{t}} \quad\left(1 \leq i_{1} \leq \cdots \leq i_{t} \leq n\right) .
$$

We define $Y(\lambda)$ to be the set of all reduced proper Young walls in $F(\lambda)$ satisfying the following conditions:

(Y1) For each $k=1, \cdots, t$, we have $Y_{\omega_{i_{k}}}^{+} \subset\left|Y_{\omega_{i_{k}}}^{-}\right|$.

(Y2) For each $k=1, \cdots, t-1$, we have $Y^{\omega_{i_{k}}} \subset Y^{\omega_{i_{k+1}}}$ in $\bar{Y}_{\omega_{i_{k}}+\omega_{i_{k+1}}}$.

(Y3) For each $k=1, \cdots, t-1$, we have $\left|Y_{\left(\omega_{i_{k}}, \omega_{i_{k+1}}\right)}^{-}\right| \subset Y_{\left(\omega_{i_{k}}, \omega_{i_{k+1}}\right.}^{+}$).

(Y4) For each $k=1, \cdots, t-1$, if $\bar{Y}_{\omega_{i_{k}}+\omega_{i_{k+1}}}$ satisfies (C1), then we have

$$
Y_{\omega_{i_{k}}}^{+}(a ; p, q) \subset\left|Y_{\omega_{i_{k}}}^{-}(a ; p, q)\right|, \quad Y_{\omega_{i_{k+1}}}^{+}(a ; p, q) \subset\left|Y_{\omega_{i_{k+1}}}^{-}(a ; p, q)\right| .
$$

Then there is an isomorphism of $U_{q}\left(C_{n}\right)$-crystals

$$
Y(\lambda) \stackrel{\sim}{\longrightarrow} B(\lambda) \text { given by } H_{\lambda} \longmapsto u_{\lambda}
$$

where $u_{\lambda}$ is the highest weight vector in $B(\lambda)$.

Theorem 2.9. Let $\lambda \in P^{+}$be a dominant integral weight for $\mathfrak{g}=B_{n}$, and write

$$
\begin{aligned}
& \lambda=\omega_{i_{1}}+\cdots+\omega_{i_{t}} \quad\left(1 \leq i_{1} \leq \cdots \leq i_{t} \leq n\right) \quad \text { or } \\
& \lambda=\omega_{i_{1}}+\cdots+\omega_{i_{t}}+\lambda_{n} \quad\left(1 \leq i_{1} \leq \cdots \leq i_{t} \leq n\right) .
\end{aligned}
$$

We define $Y(\lambda)$ to be the set of all reduced proper Young walls in $F(\lambda)$ satisfying the following conditions:

(Y1) For each $k=1, \cdots, t$, we have $Y_{\omega_{i_{k}}}^{+} \subset\left|Y_{\omega_{i_{k}}}^{-}\right|$.

(Y2) For each $k=1, \cdots, t-1$, we have

$$
Y^{\omega_{i_{k}}} \subset Y^{\omega_{i_{k+1}}} \text { in } \bar{Y}_{\omega_{i_{k}}+\omega_{i_{k+1}}} \text { and } Y^{\omega_{i_{t}}} \subset Y^{\lambda_{n}} \text { in } \bar{Y}_{\omega_{i_{t}}+\lambda_{n}} \text {. }
$$

(Y3) For each $k=1, \cdots, t-1$, we have

$$
\left|Y_{\left(\omega_{i_{k}}, \omega_{i_{k+1}}\right)}^{-}\right| \subset Y_{\left(\omega_{i_{k}}, \omega_{i_{k+1}}\right)}^{+}, \quad\left|Y_{\left(\omega_{i_{t}}, \lambda_{n}\right)}^{-}\right| \subset Y_{\left(\omega_{i_{t}}, \lambda_{n}\right)}^{+} .
$$

(Y4) For each $k=1, \cdots, t-1$, if $\bar{Y}_{\omega_{i_{k}}+\omega_{i_{k+1}}}$ or $\bar{Y}_{\omega_{i_{t}}+\lambda_{n}}$ satisfies $(\mathbf{C 1})$, then we have

$$
\begin{aligned}
& Y_{\omega_{i_{k}}}^{+}(a ; p, q) \subset\left|Y_{\omega_{i_{k}}}^{-}(a ; p, q)\right|, \quad Y_{\omega_{i_{k+1}}}^{+}(a ; p, q) \subset\left|Y_{\omega_{i_{k+1}}}^{-}(a ; p, q)\right|, \\
& Y_{\omega_{i_{t}}}^{+}(a ; p, q) \subset\left|Y_{\omega_{i_{t}}}^{-}(a ; p, q)\right| .
\end{aligned}
$$

Then there is an isomorphism of crystal graphs for $U_{q}\left(B_{n}\right)$-modules

$$
Y(\lambda) \stackrel{\sim}{\longrightarrow} B(\lambda) \text { given by } H_{\lambda} \longmapsto u_{\lambda}
$$

where $u_{\lambda}$ is the highest weight vector in $B(\lambda)$.

Remark 2.10. If $\lambda=\lambda_{n}$, then $Y\left(\lambda_{n}\right)=F\left(\lambda_{n}\right)$, the set of all reduced proper Young walls lying between $H_{\lambda_{n}}$ and $L_{\lambda_{n}}$.

Example 2.11. Let $\mathfrak{g}=C_{3}$ and $\lambda=\omega_{2}+\omega_{3}$. Then, in Figure 11, the first Young wall belongs to $Y(\lambda)$ but the second one and the third one do not. The second one does not satisfy (Y1) and the third one does not satisfy (Y2). Here, in the second Young wall, the shaded parts represent $L_{\omega_{3}}^{ \pm}$, and in the third Young wall, the shaded parts represent $L^{\omega_{2}}$ and $L^{\omega_{3}}$. 

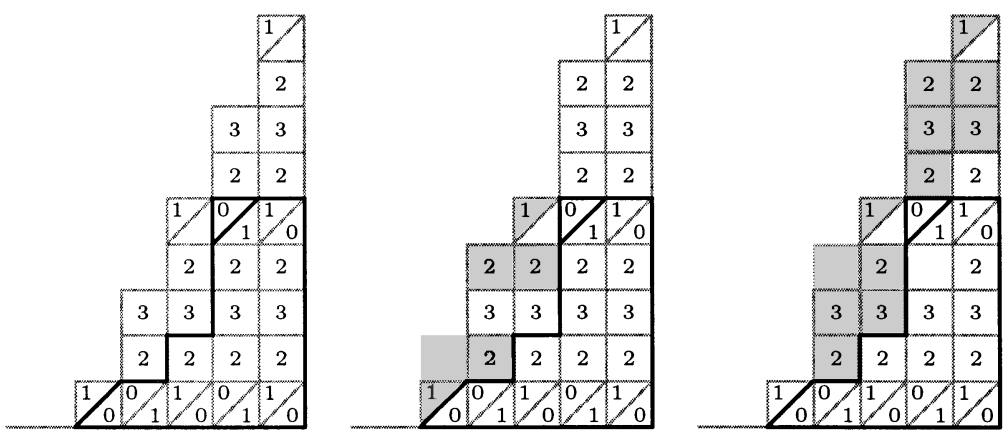

Figure 11.
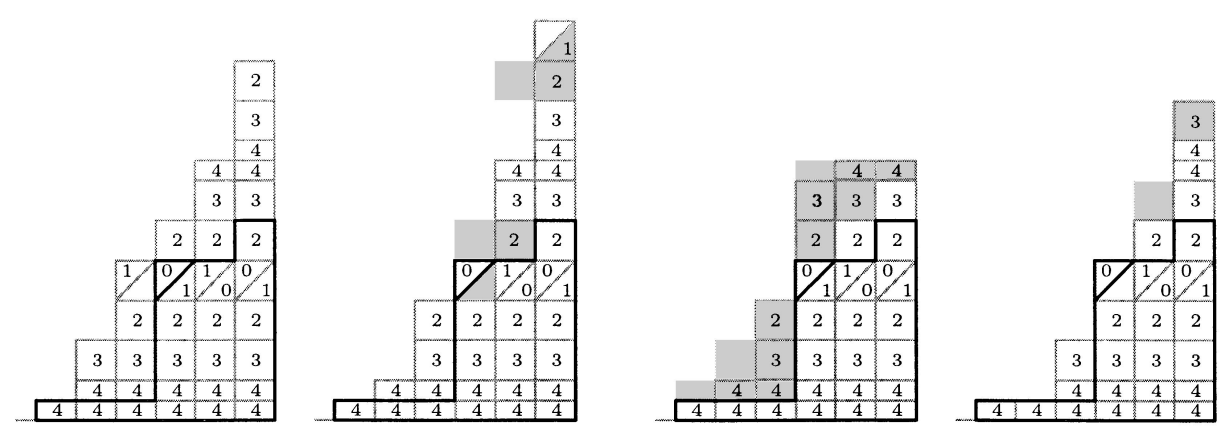

FigURE 12.

Example 2.12. Let $\mathfrak{g}=B_{4}$ and $\lambda=\omega_{3}+\lambda_{4}$. Then, in Figure 12, the first Young wall belongs to $Y(\lambda)$, but the other ones do not. They do not satisfy the conditions (Y1), (Y3) and (Y4), respectively. Here, the shaded parts represent $L_{\omega_{3}}^{ \pm}, L_{\left(\omega_{3}, \lambda_{4}\right)}^{ \pm}$ and $L_{\omega_{3}}^{ \pm}(3 ; 2,1)$ in the second, third and fourth Young walls, respectively.

Finally, we focus on the case $\mathfrak{g}=D_{n}$. If $\bar{Y}_{\omega_{i_{k}}}$ of $Y \in F(\lambda)$ contains a row consisting of $n$-blocks and $(n-1)$-blocks, which will be called the $(n-1, n)$-row, then we define the walls $Y_{\omega_{i_{k}}}^{ \pm}$and $\left|Y_{\omega_{i_{k}}}^{-}\right|$as in the case of $\mathfrak{g}=C_{n}$ or $B_{n}$.

Example 2.13. If $\mathfrak{g}=D_{5}, \lambda=\omega_{6}$ and

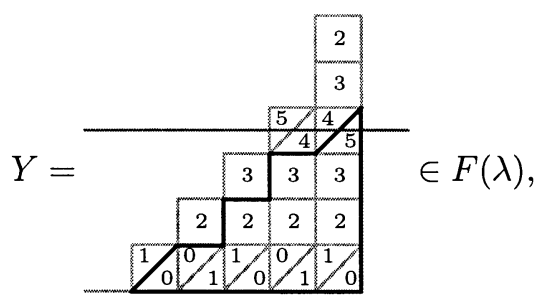


then we have

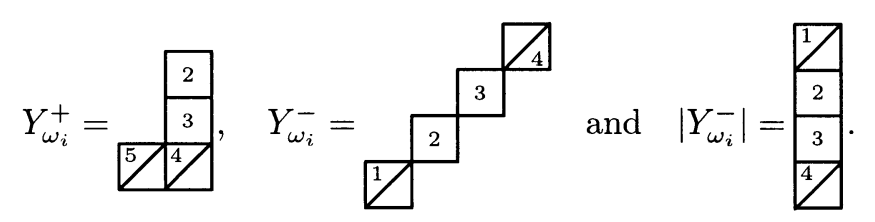

Consider $\bar{L}_{\omega_{i_{k}}+\omega_{i_{k+1}}}, \bar{L}_{\omega_{i_{t}}+\lambda_{n}}$ or $\bar{L}_{\omega_{i_{t}}+\lambda_{n-1}}$ of $Y \in F(\lambda)$. As we can see from Figure $13, \bar{L}_{\omega_{i_{k}}+\omega_{i_{k+1}}}$ contains two $(n-1, n)$-rows above $\bar{H}_{\omega_{i_{k}}+\omega_{i_{k+1}}}$. We denote by $b_{L}$ the left-most blocks in the upper $(n-1, n)$-row and $b_{R}$ the blocks lying in the $(i-2)$-th column to the right of $b_{L}$. Then the blocks $b_{L}, b_{R}$ and the block $b$ lying in the $(i-2)$-th row below $b_{L}$ form a right isosceles triangle. We denote by $L_{\left(\omega_{i_{k}}, \omega_{i_{k+1}}\right)}^{-}$the part of $\bar{L}_{\omega_{i_{k}}+\omega_{i_{k+1}}}$ consisting of this right isosceles triangle. Note that the size of $L_{\left(\omega_{i_{k}}, \omega_{i_{k+1}}\right)}^{-}$in the case of $D_{n}$ is smaller than that of $L_{\left(\omega_{i_{k}}, \omega_{i_{k+1}}\right)}^{-}$in the case of $C_{n}$ or $B_{n}$.

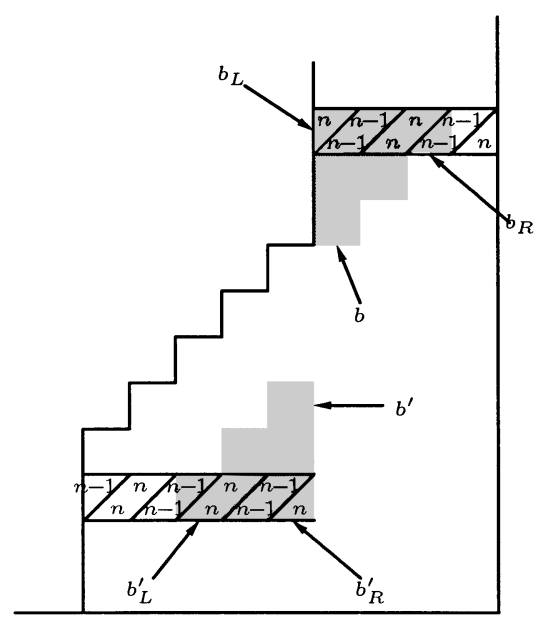

FiguRE 13.

Similarly, let $b_{R}^{\prime}$ be the right-most blocks in the lower $(n-1, n)$-row outside the highest weight vector $\bar{H}_{\omega_{i_{k}}+\omega_{i_{k+1}}}$ and let $b_{L}^{\prime}$ be the blocks lying in the $(i-2)$-th column to the left of $b_{R}^{\prime}$. Then the blocks $b_{R}^{\prime}, b_{L}^{\prime}$ and the block $b^{\prime}$ lying in the $(i-2)$ th row above $b_{R}^{\prime}$ form another right isosceles triangle. We denote by $L_{\left(\omega_{i_{k}}, \omega_{i_{k+1}}\right)}^{+}$the part of $\bar{L}_{\omega_{i_{k}}+\omega_{i_{k+1}}}$ consisting of this right isosceles triangle. Note that $L_{\left(\omega_{i_{k}}, \omega_{i_{k+1}}\right)}^{-}$ and $L_{\left(\omega_{\left.i_{k}, \omega_{i_{k+1}}\right)}^{+}\right.}^{+}$are of the same size with each base of length $i-1$. Now, we can also define $L_{\left(\omega_{i_{t}}, \lambda_{n-1}\right)}^{ \pm}$and $L_{\left(\omega_{i_{t}}, \lambda_{n}\right)}^{ \pm}$in a similar way, and set

$$
\begin{aligned}
& Y_{\left(\omega_{i_{k}}, \omega_{i_{k+1}}\right)}^{-}=Y \cap L_{\left(\omega_{i_{k}}, \omega_{i_{k+1}}\right)}^{-}, \quad Y_{\left(\omega_{i_{k}}, \omega_{i_{k+1}}\right)}^{+}=Y \cap L_{\left(\omega_{i_{k}}, \omega_{i_{k+1}}\right)}^{+}, \\
& Y_{\left(\omega_{i_{t}}, \lambda_{n-1}\right)}^{ \pm}=Y \cap L_{\left(\omega_{i_{t}}, \lambda_{n-1}\right)}^{ \pm}, \quad Y_{\left(\omega_{i_{t}}, \lambda_{n}\right)}^{ \pm}=Y \cap L_{\left(\omega_{i_{t}}, \lambda_{n}\right)}^{ \pm} .
\end{aligned}
$$


As usual, let $\left|Y_{\left(\omega_{i_{k}}, \omega_{i_{k+1}}\right)}^{-}\right|$(resp. $\left|Y_{\left(\omega_{i_{t}}, \lambda_{n-1}\right)}^{-}\right|$and $\left.\left|Y_{\left(\omega_{i_{t}}, \lambda_{n}\right)}^{-}\right|\right)$be the wall obtained by reflecting $Y_{\left(\omega_{i_{k}}, \omega_{i_{k+1}}\right)}^{-}\left(\right.$resp. $Y_{\left(\omega_{i_{t}}, \lambda_{n-1}\right)}^{-}$and $\left.Y_{\left(\omega_{i_{t}}, \lambda_{n}\right)}^{-}\right)$with respect to the $(n-1, n)$ row and shifting the blocks to the right as much as possible.

Example 2.14. If $\mathfrak{g}=D_{7}, \lambda=\omega_{5}+\lambda_{6}$, and

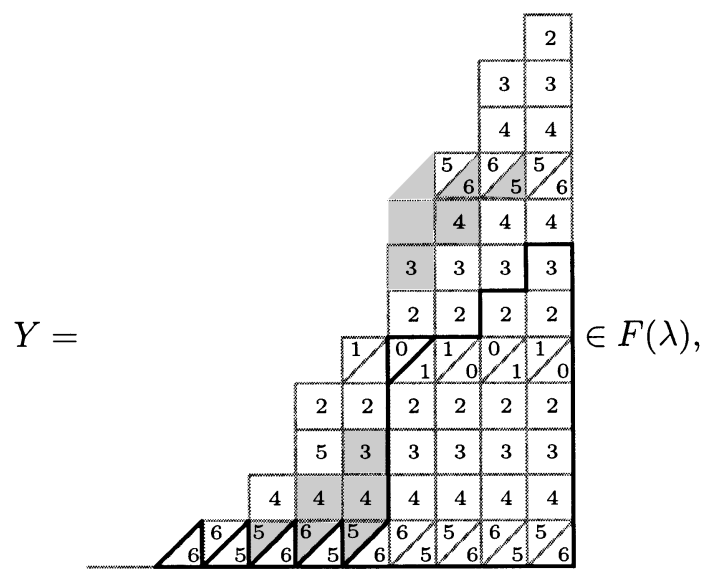

then we have

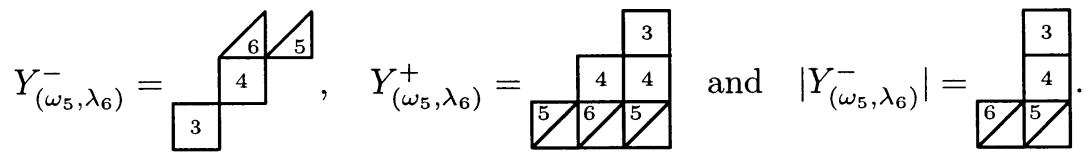

Here, the shaded parts represent $L_{\left(\omega_{5}, \lambda_{6}\right)}^{-}$and $L_{\left(\omega_{5}, \lambda_{6}\right)}^{+}$, respectively.

Assume that $\bar{Y}_{\omega_{i_{k}}+\omega_{i_{k+1}}}, \bar{Y}_{\omega_{i_{t}}+\lambda_{n}}$ or $\bar{Y}_{\omega_{i_{t}}+\lambda_{n-1}}$ of $Y \in F(\lambda)$ satisfies (C1). Then we can define $L_{\omega_{i_{k}}}^{ \pm}(a ; p, q), L_{\omega_{i_{k+1}}}^{ \pm}(a ; p, q), Y_{\omega_{i_{k}}}^{ \pm}(a ; p, q), Y_{\omega_{i_{k+1}}}^{ \pm}(a ; p, q)$ and $\left|Y_{\omega_{i_{k+1}}}^{ \pm}(a ; p, q)\right|$ as in the case of $\mathfrak{g}=C_{n}$ or $B_{n}$.

Now, suppose that $\bar{Y}_{\omega_{i_{k}}+\omega_{i_{k+1}}}, \bar{Y}_{\omega_{i_{t}}+\lambda_{n}}$ or $\bar{Y}_{\omega_{i_{t}}+\lambda_{n-1}}$ of $Y \in F(\lambda)$ have the configuration in Figure 14. That is, the top of the $p$-th column of $\bar{Y}_{\omega_{i_{k}}}$ from the right is $\frac{n}{n-2},\left|\frac{n}{n-2}\right|, \frac{n}{n-2}$ or $\frac{n-1}{n-2}$. In the case of $\frac{n}{n-2}$ or $\frac{\mid n}{n-2}\left(\right.$ resp. $\frac{n-2}{n-2}$ or $\left.\frac{n-1}{n-2}\right)$, the top of the $q$-th column of $\bar{Y}_{\omega_{i_{k+1}}}$ from the right is $\sum_{n-2}^{n}$ or $\frac{Z_{n-2}}{n}$ (resp. $\frac{n=1}{\sum_{n-2}}$ or $\frac{n-1}{n-2}$ ) when $q-p$ is odd and the top of the $q$-th column of $\bar{Y}_{\omega_{i_{k+1}}}$ from the right is $\left|\frac{n-1}{n-2}\right|$ or $\frac{n-1}{n-2}$ (resp. $\sqrt[n-2]{n}$ or $\frac{n}{n-2}$ ) when $q-p$ is even.

We define $L_{\omega_{i_{k}}}(n-1, n ; p, q)$ to be the parallelogram formed by the $(n-q)$ block and $(n-q+p-1)$-block in the $q$-th column, and the $(n-i)$-block and $(n-i+p-1)$-block in the $i$-th column lying below the $(n-1, n)$-row. We will denote by $L_{\omega_{i_{k+1}}}(n-1, n ; p, q)$ the parallelogram formed by the $(n-q)$-block and $(n-i)$-block in the first column, and the $(n-q+p-1)$-block and $(n-i+p-1)$ block in the $p$-th column lying above the $(n-1, n)$-row. Similarly, we can define the parallelograms $L_{\omega_{i_{t}}}(n-1, n ; p, q), L_{\lambda_{n}}(n-1, n ; p, q)$ and $L_{\lambda_{n-1}}(n-1, n ; p, q)$. The 
(C2)

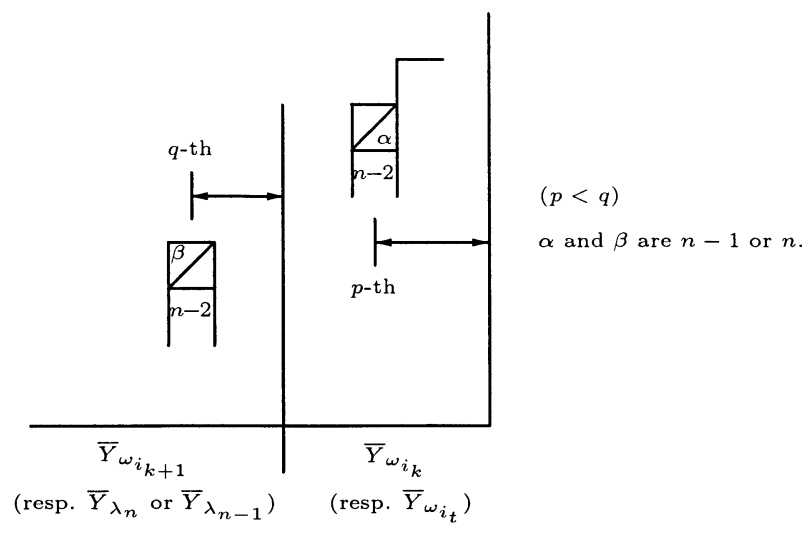

Figure 14.

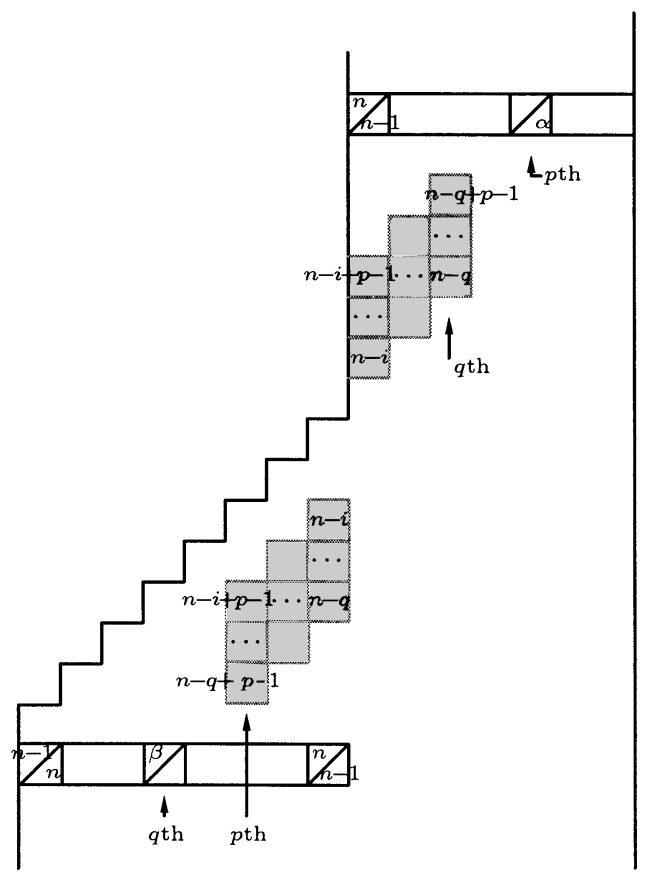

Figure 15.

shaded parts in Figure 15 represent $L_{\omega_{i_{k}}}(n-1, n ; p, q)$ and $L_{\omega_{i_{k+1}}}(n-1, n ; p, q)$. Here, $\alpha$ and $\beta$ are $n$ or $n-1$ in the hypothesis.

We define

$$
\begin{aligned}
Y_{\omega_{i_{k}}}(n-1, n ; p, q) & =L_{\omega_{i_{k}}}(n-1, n ; p, q) \cap Y, \\
Y_{\omega_{i_{k+1}}}(n-1, n ; p, q) & =L_{\omega_{i_{k+1}}}(n-1, n ; p, q) \cap Y, \\
Y_{\omega_{i_{t}}}(n-1, n ; p, q) & =L_{\omega_{i_{t}}}(n-1, n ; p, q) \cap Y, \\
Y_{\lambda_{n-1}}(n-1, n ; p, q) & =L_{\lambda_{n-1}}(n-1, n ; p, q) \cap Y, \\
Y_{\lambda_{n}}(n-1, n ; p, q) & =L_{\lambda_{n}}(n-1, n ; p, q) \cap Y,
\end{aligned}
$$


and let $\left|Y_{\omega_{i_{k}}}(n-1, n ; p, q)\right|$ be the wall obtained by reflecting $Y_{\omega_{i_{k}}}(n-1, n ; p, q)$ with respect to the $(n-1, n)$-arrow and shifting the blocks to the right as much as possible and let $Y_{\omega_{i_{k+1}}}^{t}(n-1, n ; p, q)$ be the wall obtained by shifting the blocks of $Y_{\omega_{i_{k+1}}}(n-1, n ; p, q)$ to the right as much as possible.

Example 2.15. If $\mathfrak{g}=D_{8}, \lambda=\omega_{5}+\omega_{6}$ and

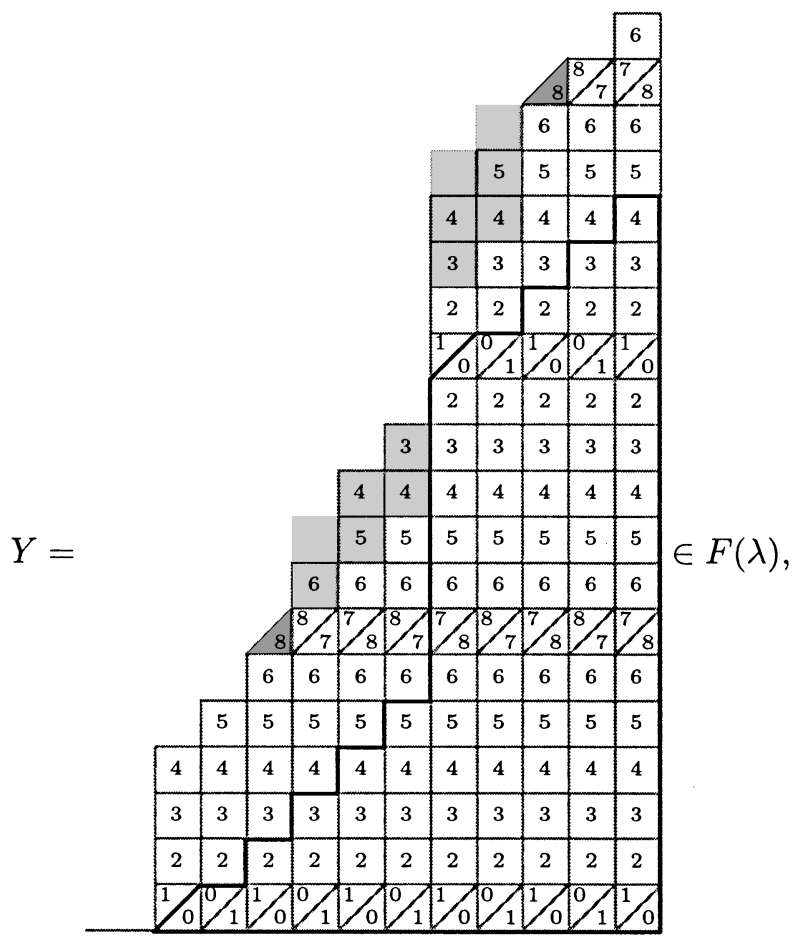

then we have

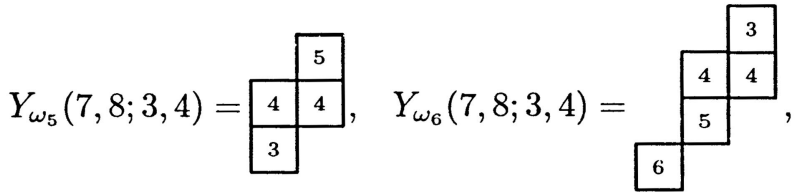

$$
\begin{aligned}
& \left|Y_{\omega_{5}}(7,8 ; 3,4)\right|=\begin{array}{|l|l|}
\hline 4 & 3 \\
\hline 4 & 4 \\
\hline 5
\end{array} \text { and } Y_{\omega_{6}}^{t}(7,8 ; 3,4)=\begin{array}{|l|l|}
\hline 4 & 3 \\
\hline 4 & 5 \\
\hline
\end{array}
\end{aligned}
$$

Here, the shaded parts represent $L_{\omega_{5}}(7,8 ; 3,4)$ and $L_{\omega_{6}}(7,8 ; 3,4)$.

Theorem 2.16. Let $\lambda \in P^{+}$be a dominant integral weight for $\mathfrak{g}=D_{n}$ and write

$$
\lambda=\omega_{i_{1}}+\cdots+\omega_{i_{t}}+b_{1} \lambda_{n-1}+b_{2} \lambda_{n}
$$

where $1 \leq i_{1} \leq \cdots \leq i_{t} \leq n+1$ and $\left(b_{1}, b_{2}\right)=(1,0)$ or $(0,1)$. 
Define $Y(\lambda)$ to be the set of all reduced proper Young walls in $F(\lambda)$ satisfying the following conditions:

(Y1) For each $k=1, \cdots, t$, we have $Y_{\omega_{i_{k}}}^{+} \subset\left|Y_{\omega_{i_{k}}}^{-}\right|$.

(Y2) For each $k=1, \cdots, t-1$, we have

$Y^{\omega_{i_{k}}} \subset Y^{\omega_{i_{k+1}}}$ in $\bar{Y}_{\omega_{i_{k}}+\omega_{i_{k+1}}}, Y^{\omega_{i_{t}}} \subset Y^{\lambda_{n-1}}$ in $\bar{Y}_{\omega_{i_{t}}+\lambda_{n-1}}, Y^{\omega_{i_{t}}} \subset Y^{\lambda_{n}}$ in $\bar{Y}_{\omega_{i_{t}}+\lambda_{n}}$.

(Y3) For each $k=1, \cdots, t-1$, we have

$$
\left|Y_{\left(\omega_{i_{k}}, \omega_{i_{k+1}}\right)}^{-}\right| \subset Y_{\left(\omega_{i_{k}}, \omega_{i_{k+1}}\right)}^{+}, \quad\left|Y_{\left(\omega_{i_{t}}, \lambda_{n-1}\right)}^{-}\right| \subset Y_{\left(\omega_{i_{t}}, \lambda_{n-1}\right)}^{+}, \quad\left|Y_{\left(\omega_{i_{t}}, \lambda_{n}\right)}^{-}\right| \subset Y_{\left(\omega_{i_{t}}, \lambda_{n}\right)}^{+} .
$$

(Y4) For each $k=1, \cdots, t-1$, if $\bar{Y}_{\omega_{i_{k}}+\omega_{i_{k+1}}}, \bar{Y}_{\omega_{i_{t}}+\lambda_{n-1}}$ or $\bar{Y}_{\omega_{i_{t}}+\lambda_{n}}$ satisfies (C1), then we have

$$
\begin{aligned}
& Y_{\omega_{i_{k}}}^{+}(a ; p, q) \subset\left|Y_{\omega_{i_{k}}}^{-}(a ; p, q)\right|, \quad Y_{\omega_{i_{k+1}}}^{+}(a ; p, q) \subset\left|Y_{\omega_{i_{k+1}}}^{-}(a ; p, q)\right|, \\
& Y_{\omega_{i_{t}}}^{+}(a ; p, q) \subset\left|Y_{\omega_{i_{t}}}^{-}(a ; p, q)\right| .
\end{aligned}
$$

(Y5) For each $k=1, \cdots, t-1$, if $\bar{Y}_{\omega_{i_{k}}+\omega_{i_{k+1}}}, \bar{Y}_{\omega_{i_{t}}+\lambda_{n-1}}$ or $\bar{Y}_{\omega_{i_{t}}+\lambda_{n}}$ satisfies (C2), then we have

$$
\begin{aligned}
& \left|Y_{\omega_{i_{k}}}(n-1, n ; p, q)\right| \subset Y_{\omega_{i_{k+1}}}^{t}(n-1, n ; p, q), \\
& \left|Y_{\omega_{i_{t}}}(n-1, n ; p, q)\right| \subset Y_{\lambda_{n-1}}^{t}(n-1, n ; p, q), \\
& \left|Y_{\omega_{i_{t}}}(n-1, n ; p, q)\right| \subset Y_{\lambda_{n}}^{t}(n-1, n ; p, q) .
\end{aligned}
$$

Then there exists an isomorphism of $U_{q}\left(D_{n}\right)$-crystals

$$
Y(\lambda) \stackrel{\sim}{\longrightarrow} B(\lambda) \quad \text { given by } H_{\lambda} \longmapsto u_{\lambda},
$$

where $u_{\lambda}$ is the highest weight vector in $B(\lambda)$.

Example 2.17. Let $\mathfrak{g}=D_{4}$ and $\lambda=\omega_{3}+\lambda_{4}$. Then, in Figure 16, the first Young wall belongs to $Y(\lambda)$, but the other ones do not. They do not satisfy the conditions (Y1), (Y3), (Y4) and (Y5), respectively. Here, the shaded parts represent $L_{\omega_{3}}^{ \pm}, L_{\left(\omega_{3}, \lambda_{4}\right)}^{ \pm}, L_{\omega_{3}}^{ \pm}(2 ; 3,1)$, and $L_{\omega_{3}}(3,4 ; 1,2)$ and $L_{\lambda_{4}}(3,4 ; 1,2)$ in the second, third, fourth and fifth Young walls, respectively.

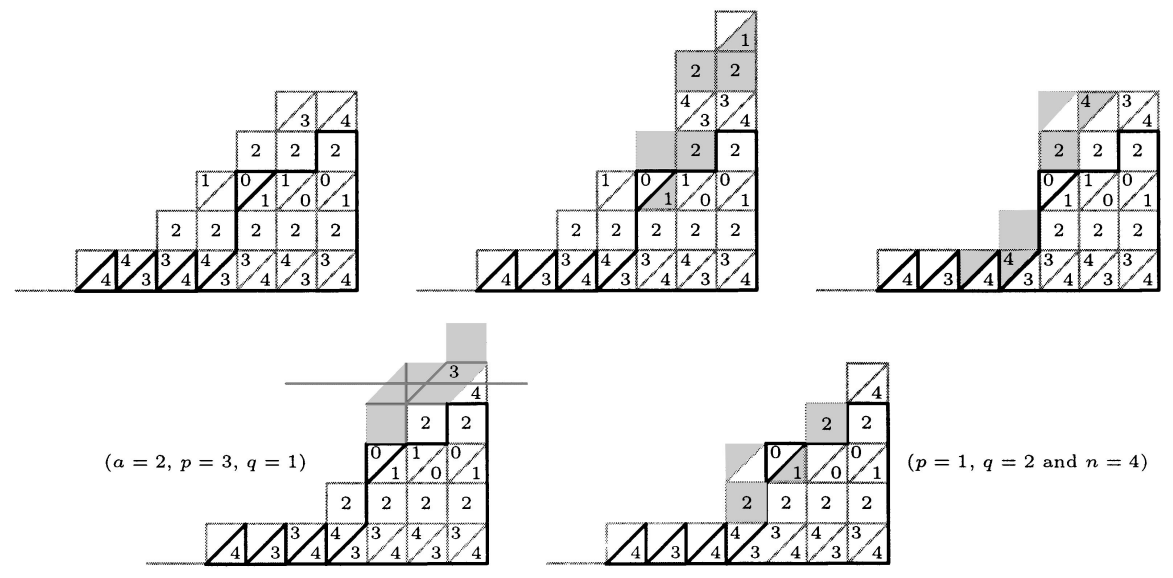

Figure 16. 


\section{The PROOF OF THE MAIN THEOREM}

In this section, we will give a proof of our main theorems. In fact, we will only prove the case $\mathfrak{g}=B_{n}$ because the remaining cases can be proved in a similar manner. Observe that it suffices to prove the following statements:

(1) For all $i=1, \cdots, n$, we have

$$
\tilde{e}_{i} Y(\lambda) \subset Y(\lambda) \cup\{0\}, \quad \tilde{f}_{i} Y(\lambda) \subset Y(\lambda) \cup\{0\} .
$$

(2) If $Y \in Y(\lambda)$ satisfies $\tilde{e}_{i} Y=0$ for all $i=1, \cdots, n$, then $Y=H_{\lambda}$.

The proof of Theorem 2.9. We first prove the statement (1). Let $Y \in Y(\lambda)$ and suppose that $\tilde{f}_{i} Y \neq 0$ but $\tilde{f}_{i} Y \notin Y(\lambda)$ for some $i \in I$. Then $\tilde{f}_{i} Y$ would violate at least one of the conditions (Y1)-(Y4).

(Case 1) Suppose $\tilde{f}_{i} Y$ does not satisfy (Y1). Then there is an $i$-admissible slot in some $Y_{\omega_{i_{k}}}^{+}$, where an $i$-block can be added to get $\tilde{f}_{i} Y$ such that $\left(\tilde{f}_{i} Y\right)_{\omega_{i_{k}}}^{+} \not$ $\left(\tilde{f}_{i} Y\right)_{\omega_{i_{k}}}^{-}$. Note that $i \neq n$ because $\left(\tilde{f}_{n} Y\right)_{\omega_{i_{k}}}^{ \pm}=Y_{\omega_{i_{k}}}^{ \pm}$for all $k=1, \cdots, t$. For simplicity, we denote by $N_{j}^{ \pm}$the number of $j$-blocks in $Y_{\omega_{i_{k}}}^{ \pm}$. Then $N_{j}^{+} \leq N_{j}^{-}$for all $j=1, \cdots, n-1$ because $Y_{\omega_{i_{k}}}^{+} \subset Y_{\omega_{i_{k}}}^{-}$. Since $Y$ is proper, we have

$$
N_{i-1}^{+}=N_{i}^{+} \text {and } N_{i+1}^{+}=N_{i}^{+}+1 \text {. }
$$

Moreover, since $Y_{\omega_{i_{k}}}^{+} \subset Y_{\omega_{i_{k}}}^{-}$but $\left(\tilde{f}_{i} Y\right)_{\omega_{i_{k}}}^{+} \not \subseteq\left(\tilde{f}_{i} Y\right)_{\omega_{i_{k}}}^{-}$, we can deduce $N_{i}^{-}=N_{i}^{+}=$

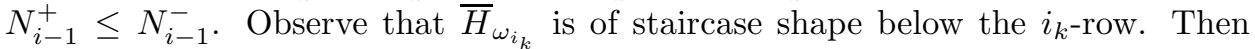
we have $N_{i+1}^{-} \leq N_{i}^{-}+1$. But we know that $N_{i}^{-}+1=N_{i}^{+}+1=N_{i+1}^{+} \leq N_{i+1}^{-}$. Therefore, $N_{i+1}^{-}=N_{i}^{-}+1$ and $Y$ must have the form shown in Figure 17. That is, there is another $i$-admissible slot in $Y_{\omega_{i_{k}}}^{-}$. Then, by the tensor product rule for the Kashiwara operators, $\tilde{f}_{i}$ would have acted on the $i$-admissible slot in $Y_{\omega_{i_{k}}}^{-}$, not on the one in $Y_{\omega_{i_{k}}}^{+}$, which is a contradiction. Hence, $\tilde{f}_{i} Y$ must satisfy the condition (Y1).

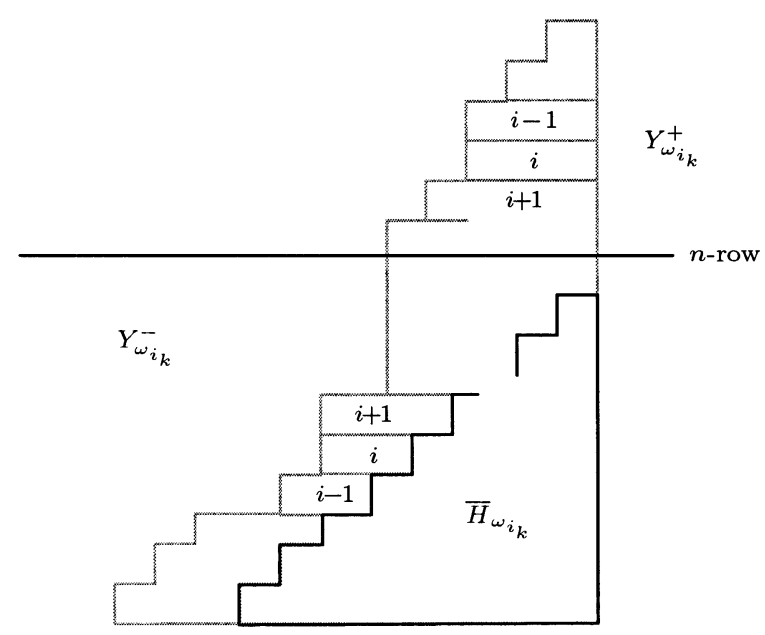

FIGURE 17. 

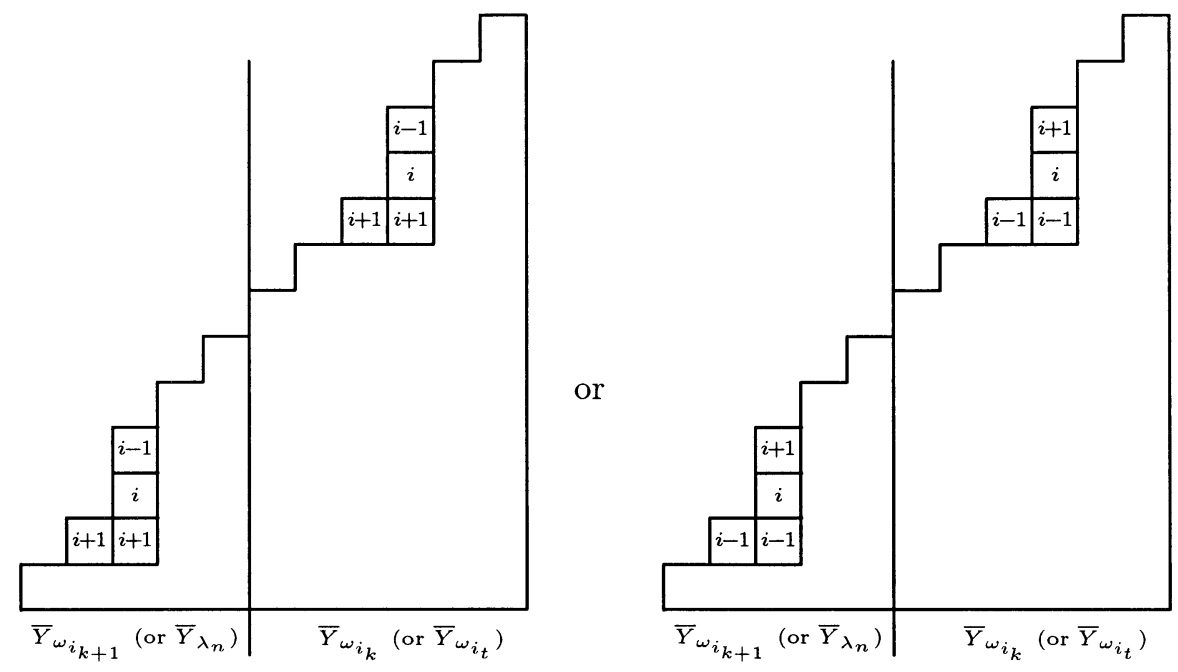

FiguRe 18.

(Case 2) Suppose $\tilde{f}_{i} Y$ does not satisfy (Y2). Then there exists an $i$-admissible slot in some $Y^{\omega_{i_{k}}}$ (or $Y^{\omega_{i_{t}}}$ ), where an $i$-block can be added to get $\tilde{f}_{i} Y$ such that

$$
\left.\left(\tilde{f}_{i} Y\right)^{\omega_{i_{k}}} \nsubseteq\left(\tilde{f}_{i} Y\right)^{\omega_{i_{k+1}}} \quad \text { (or }\left(\tilde{f}_{i} Y\right)^{\omega_{i_{t}}} \nsubseteq\left(\tilde{f}_{i} Y\right)^{\lambda_{n}}\right) .
$$

If $1 \leq i \leq n-1$, since $Y$ is proper, $Y$ has a subwall of the form

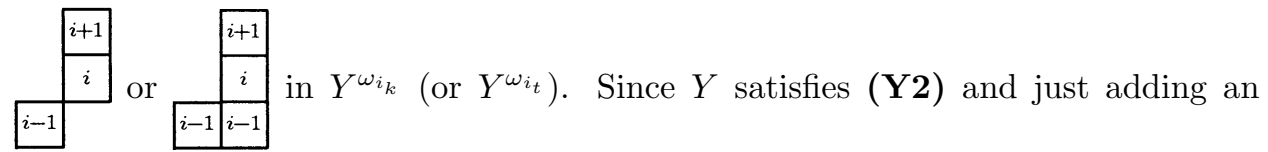
$i$-block to $Y^{\omega_{i_{k}}}$ would violate (Y2), the same part appears in $Y^{\omega_{i_{k+1}}}$ (or $Y^{\lambda_{n}}$ ), as is shown in Figure 18.

We claim that there is no removable $i$-block between these two parts. Then by the tensor product rule, $\tilde{f}_{i}$ would have acted on the $i$-admissible slot in $Y^{\omega_{i_{k+1}}}$ (or $Y^{\lambda_{n}}$ ), not on the one in $Y^{\omega_{i_{k}}}$ (or $Y^{\omega_{i}}$ ), which is a contradiction. Hence $\tilde{f}_{i} Y$ must satisfy (Y2). To prove our claim, assume first that there exists a removable $i$-block in $Y^{\omega_{i_{k}}}$. Then $Y$ must have the shape given in Figure 19. Thus $Y_{\left(\omega_{i_{k}}, \omega_{i_{k+1}}\right)}^{+}$would

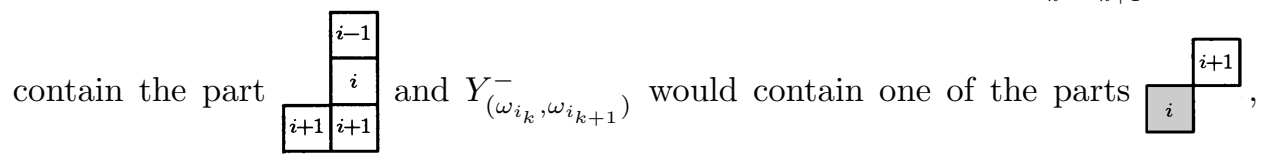

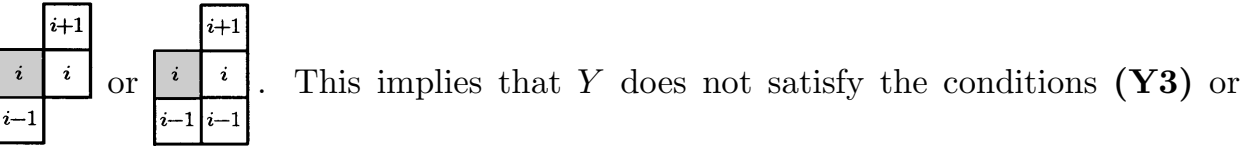
(Y4), which is a contradiction. Hence there is no removable $i$-block in $Y^{\omega_{i_{k}}}$. Next, assume that there exists a removable $i$-block in $Y^{\omega_{i}}{ }_{k+1}$. Then $Y$ must have the shape given in Figure 20. 


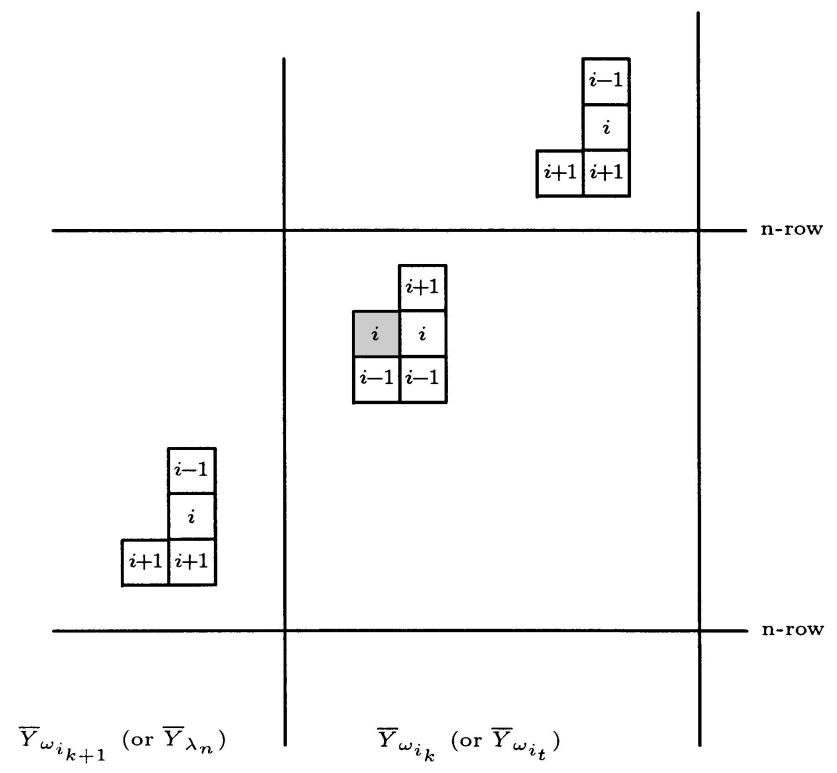

FiguRE 19.

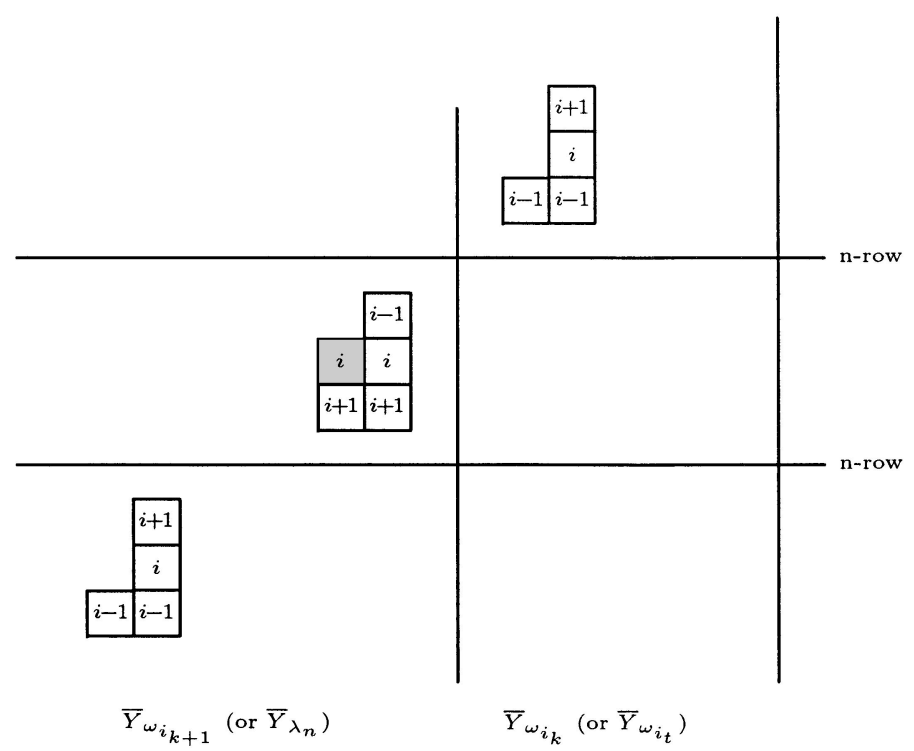

FIGURE 20.

Now, by a similar argument as above, one can see that $Y$ does not satisfy (Y3) or (Y4), which is a contradiction. Thus there is no removable $i$-block between these two parts as we claimed. 


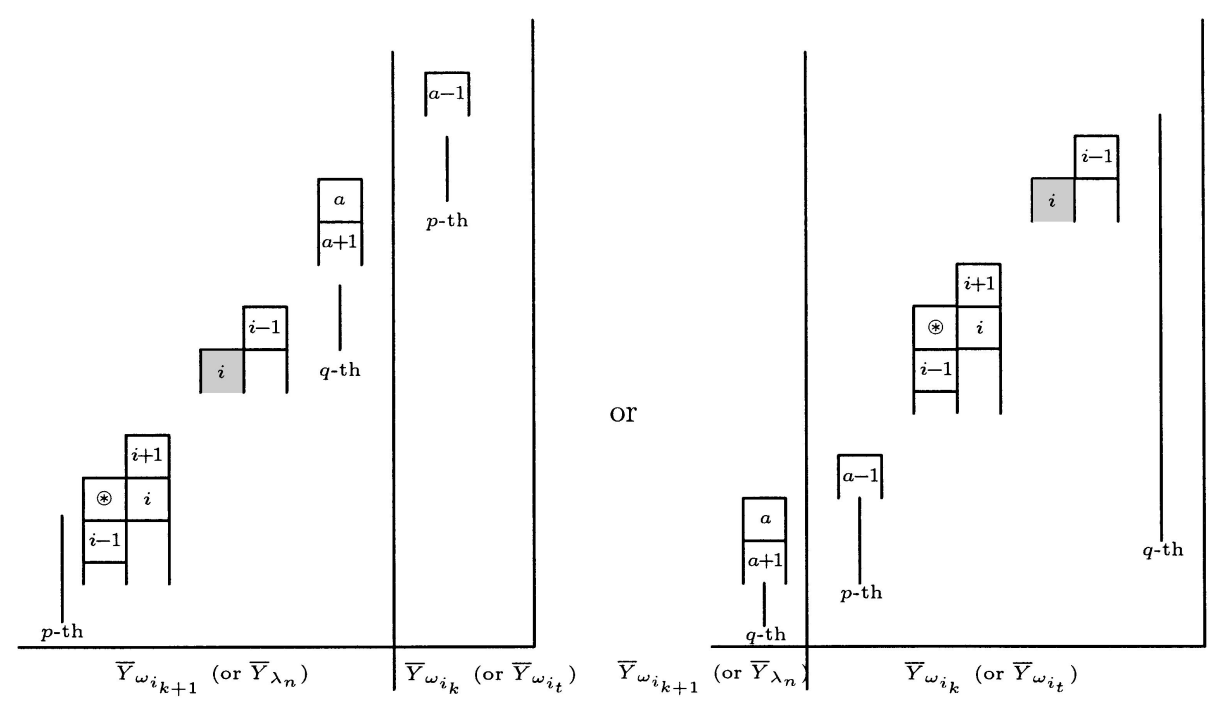

FiguRe 21.

If $i=n$, then $Y$ has a subwall of the form

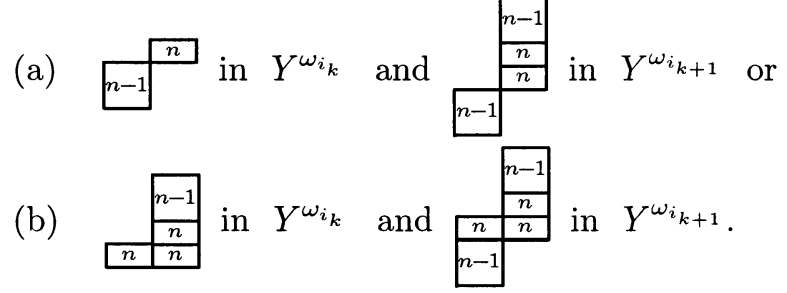

The case (b) does not occur because $Y$ would not satisfy the condition (Y3). In the case of (a), $\tilde{f}_{n}$ would have acted on the $n$-admissible slot in $Y^{\omega_{i_{k+1}}}$ not on the one in $Y^{\omega_{i_{k}}}$, which is a contradiction. Therefore, $\tilde{f}_{i} Y$ must satisfy the condition (Y2).

(Case 3) If $\tilde{f}_{i} Y$ does not satisfy (Y3), then by a similar argument to (Case 1) and (Case 2), we can derive a contradiction. Hence, $\tilde{f}_{i} Y$ must satisfy the condition (Y3).

(Case 4) Suppose that $\tilde{f}_{i} Y(1 \leq i \leq n-1)$ has the configuration (C1) but does not satisfy (Y4). (If $i=n, \tilde{f}_{n} Y$ does not have the configuration (C1) by the condition (Y3).) Then we have the following two possibilities:

(a) $Y$ has the configuration (C1), $Y$ satisfies (Y4), but $\tilde{f}_{i} Y$ does not satisfy (Y4).

(b) $Y$ does not have the configuration $(\mathbf{C 1}), \tilde{f}_{i} Y$ has the configuration (C1), but $\tilde{f}_{i} Y$ does not satisfy (Y4).

On the one hand, in the case of (a), $\tilde{f}_{i} Y$ must have the form shown in Figure 21 because $Y$ satisfies (Y4). Then $\tilde{f}_{i}$ would have acted on $\circledast$, not on $i$, which is a contradiction.

On the other hand, in the case of (b), observe that, by adding an $i$-block to $Y$, $\tilde{f}_{i} Y$ can have the configuration (C1) with $a=i$ or $a=i+1$, which is shown in 
(i)

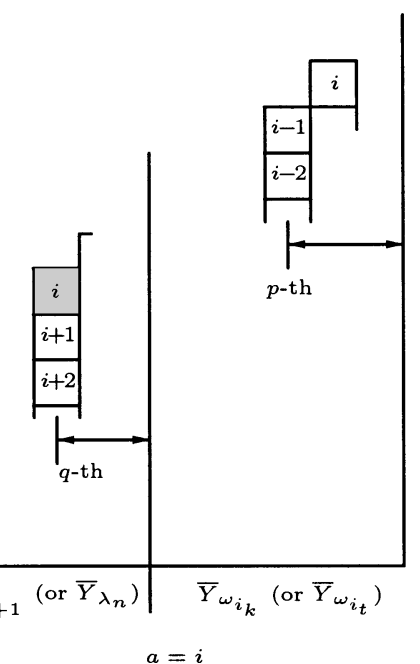

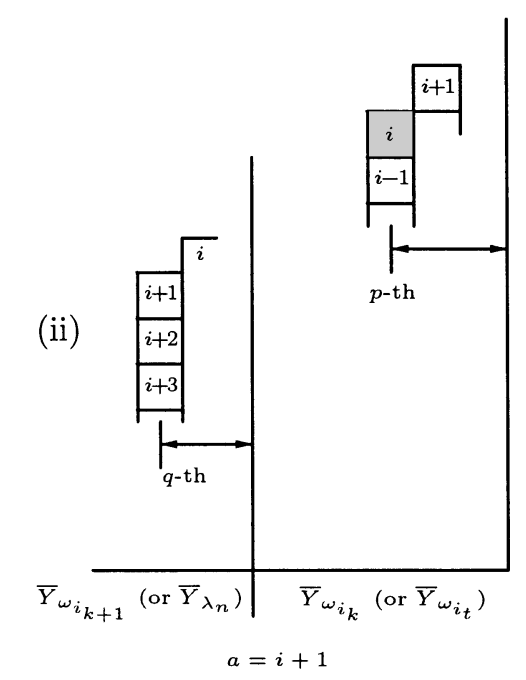

FIGURE 22.
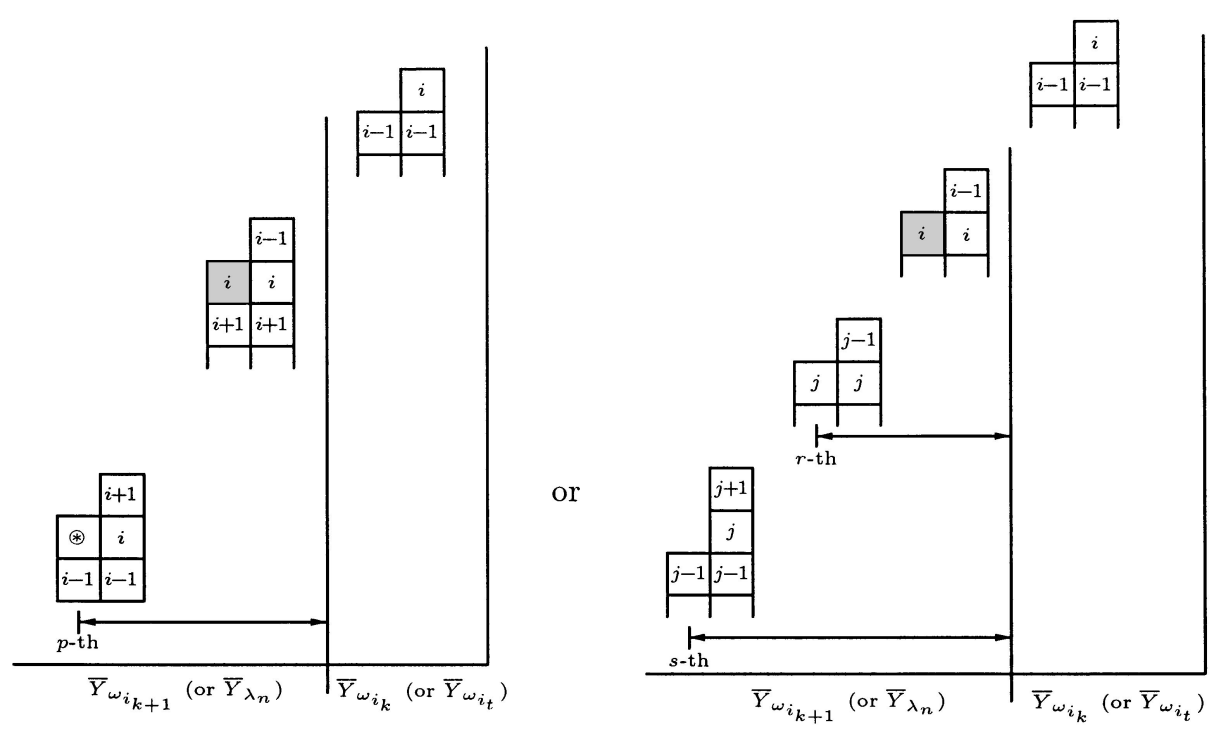

Figure 23.

Figure 22. In case (i) of this figure, if $\tilde{f}_{i} Y$ violates the condition (Y4), then $\tilde{f}_{i} Y$ must have the form shown in Figure 23. In the first case, $\tilde{f}_{i}$ would have acted on $\circledast$, not on $i$, which is a contradiction. In the second case, since $Y$ satisfies (Y1) and (Y2), we can observe that $Y$ must have the form shown in Figure 24, where $s \leq t<p, q<u<r$ and $i<k<j$. That is, $Y$ also has the configuration (C1) and does not satisfy (Y4), which is a contradiction. 


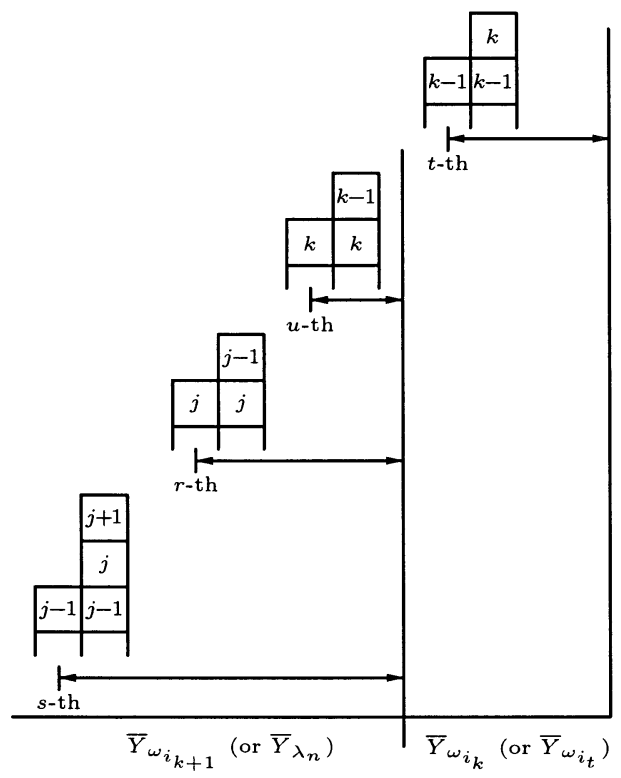

FiguRe 24.

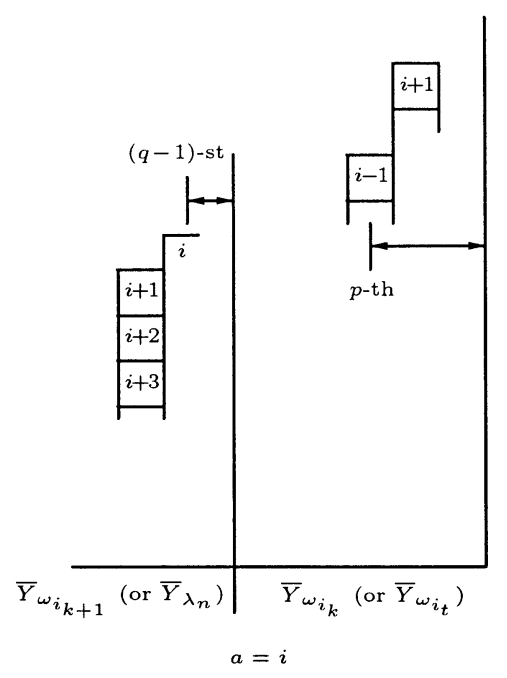

FiguRe 25.

In case (ii) of Figure 22, we note that the top of the $(q-1)$-th column in $\bar{Y}_{\omega_{i_{k+1}}}$ (or $\bar{Y}_{\lambda_{n}}$ ) must be an $i$-block by the tensor product rule for the Kashiwara operators. Then we know that $Y$ also has a configuration (C1) (see Figure 25).

But, since $Y$ satisfies the condition (Y4), adding an $i$-block to $\bar{Y}_{\omega_{i_{k}}}$ does not create a Young wall which violates the condition (Y4); i.e., the second case does not occur.

Similarly, if $Y \in Y(\lambda)$, then we can show that $\tilde{e}_{i} Y \in Y(\lambda) \cup\{0\}$. 


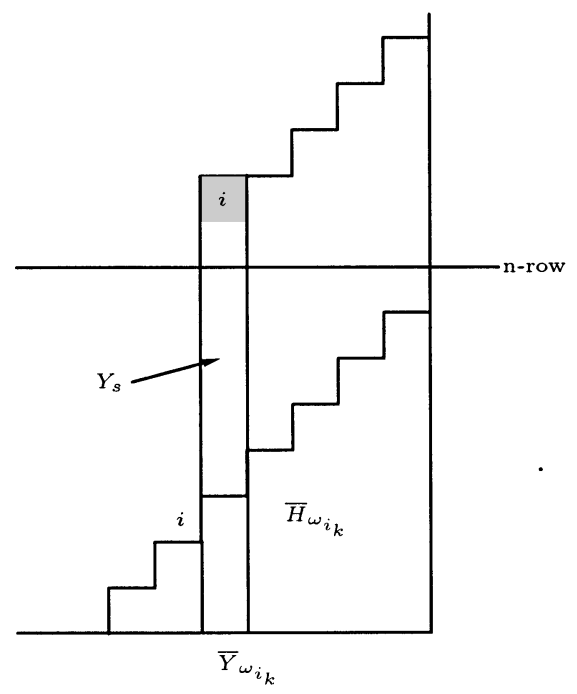

FiguRE 26.

Now, it remains to prove the statement (2). Suppose $Y \in Y(\lambda)$ and $\tilde{e}_{i} Y=0$ for all $i=1, \cdots, n$. If $Y \neq H_{\lambda}$, then there is a column in $Y$ which is higher than $H_{\lambda}$. Consider the left-most column $Y_{s}$ among them, which would belong to $\bar{Y}_{\omega_{i_{k}}}$ or $\bar{Y}_{\lambda_{n}}$. Let $i$ be the block lying in the top of the column $Y_{s}$. If there is an $i$-admissible slot to the left of $i$, then $Y$ has the form shown in Figure 26.

However, in this case, $Y_{\omega_{i_{k}}}^{+} \nsubseteq\left|Y_{\omega_{i_{k}}}^{-}\right|$, which violates the condition (Y1). Hence, there is no admissible $i$-slot to the left of $Y_{s}$, which implies $\tilde{e}_{i} Y=Y \nearrow \quad i \neq 0$, a contradiction. Therefore, $Y$ must be equal to $H_{\lambda}$.

\section{ACKNOWLEDGMENTS}

We express our sincere gratitude to Professor Georgia Benkart for her interest in this work and many valuable discussions.

\section{REFERENCES}

[1] W. Fulton, Young Tableau: with applications to representation theory and geometry, Cambridge University Press, 1997. MR 99f:05119

[2] J. Hong, S.-J. Kang, Introduction to Quantum Groups and Crystal Bases, Graduate Studies in Mathematics 42, Amer. Math. Soc., 2002. MR 2002m:17012

[3] S.-J. Kang, Crystal bases for quantum affine algebras and combinatorics of Young walls, Proc. London Math. Soc. 86 (2003), 29-69.

[4] S.-J. Kang, J.-H. Kwon, Fock space representations of quantum affine algebras and generalized Lascoux-Leclerc-Thibon algorithm, KIAS preprint M02011.

[5] S.-J. Kang, K. C. Misra, Crystal bases and tensor product decompositions of $U_{q}\left(G_{2}\right)$-modules, J. Algebra 163 (1994), 675-691. MR 95f:17013

[6] M. Kashiwara, Crystalizing the q-analogue of universal enveloping algebras, Comm. Math. Phys. 133 (1990), 249-260. MR 92b:17018

[7] $ـ$, On crystal bases of the q-analogue of universal enveloping algebras, Duke Math. J. 63 (1991), 465-516. MR 93b:17045 
[8] _ Similarity of crystal bases, in Lie Algebras and Their Representations, Seoul (1995), S.-J. Kang, M.-H. Kim, I. Lee (eds.), Contemp. Math. 194 (1996), Amer. Math. Soc., 177-186. MR 97g:17013

[9] M. Kashiwara, T. Nakashima, Crystal graphs for representations of the q-analogue of classical Lie algebras, J. Algebra 165 (1994), 295-345. MR 95c:17025

[10] J.-A. Kim, D.-U. Shin, Correspondence between Young walls and Young tableaux realizations of crystal bases for the classical Lie algebras, preprint math.QA/0303287.

[11] H. Lee, Demazure crystals of type $A_{n}$ and Young walls, KIAS preprint M02007.

[12] P. Littelmann, Crystal graphs and Young tableaux, J. Algebra 175 (1995), 65-87. MR 96h:17022

[13] _ Paths and root operators in representation theory, Ann. of Math. 142 (1995), 499525. MR 96m:17011

[14] _ A Littlewood-Richardson rule for symmetrizable Kac-Moody algebras, Invent. Math. 116 (1994), 329-346. MR 95f:17023

[15] I. G. Macdonald, Symmetric Functions and Hall Polynomials, Oxford University Press, Oxford, 2nd ed., 1995. MR 96h:05207

[16] T. Nakashima, Crystal base and a generalization of the Littlewood-Richardson rule for classical Lie algebras, Comm. Math. Phys. 154 (1993), 215-243. MR 94f:17015

School of Mathematics, Korea Institute for Advanced Study, 207-43 Cheongryangri 2 Dong, Dongdaemun-gu, Seoul 130-722, Korea

E-mail address: sjkang@kias.re.kr

Department of Mathematics, Seoul National University, Seoul 151-747, Korea

E-mail address: jakim@math.snu.ac.kr

School of Mathematics, Korea Institute for Advanced Study, 207-43 Cheongryangri 2 Dong, Dongdaemun-gu, Seoul 130-722, Korea

E-mail address: hmlee@kias.re.kr

School of Mathematics, Korea Institute for Advanced Study, 207-43 Cheongryangri 2 Dong, Dongdaemun-gu, Seoul 130-722, Korea

E-mail address: shindong@kias.re.kr 